\title{
Astrocytic Sonic Hedgehog Alleviates Intracerebral Hemorrhagic Brain Injury via Modulation of Blood-Brain Barrier Integrity
}

\author{
Gebeili Xing ${ }^{1,2+}$, Tianman Zhao ${ }^{1 \dagger}$, Xiyue Zhang ${ }^{1}, \mathrm{He} \mathrm{Li}^{1}$, Xiuping $\mathrm{Li}^{1}$, Pan Cui ${ }^{1}$, Minshu $\mathrm{Li}^{1}$, \\ Daojing $L^{3}{ }^{3}$, Nan Zhang ${ }^{1 *}$ and Wei Jiang ${ }^{1 *}$ \\ ${ }^{1}$ Department of Neurology, Tianjin Neurological Institute, Tianjin Medical University General Hospital, Tianjin, China, \\ 2Department of Neurology, Inner Mongolia People's Hospital, Hohhot, China, ${ }^{3}$ Department of Neurology, Affiliated Hospital of \\ Jining Medical University, Jining, China
}

\section{OPEN ACCESS}

Edited by:

Marco Bacigaluppi, San Raffaele Scientific Institute (IRCCS), Italy

Reviewed by: Hector Rosas-Hernandez, National Center for Toxicological Research (FDA), United States Muge Yemisci,

Hacettepe University, Turkey

*Correspondence:

Nan Zhang nkzhangnan@yeah.net Wei Jiang

jiangwei.med@gmail.com

${ }^{\dagger}$ These authors have contributed equally to this work

Specialty section:

This article was submitted to Cellular Neuropathology, a section of the journal Frontiers in Cellular Neuroscience

Received: 25 June 2020 Accepted: 08 October 2020 Published: 03 December 2020

Citation:

Xing G, Zhao T, Zhang X, Li H, Li X,

Cui P, Li M, Li D, Zhang N and Jiang W (2020) Astrocytic Sonic Hedgehog Alleviates Intracerebral

Hemorrhagic Brain Injury via Modulation of Blood-Brain Barrier Integrity. Front. Cell. Neurosci. 14:575690. doi: 10.3389/fncel.2020.575690
Background: Intracerebral hemorrhage $(\mathrm{ICH})$ is a fatal subtype of stroke that lacks effective therapy. Blood-brain barrier (BBB) damage is a hallmark of $\mathrm{ICH}$-induced brain injury that leads to edema formation, leukocytes infiltration, influx of blood components into the perihematomal (PHE) region, and eventually brain injury. Astrocytes are essential for the formation and maintenance of the BBB by providing secreted molecules that contribute to the association between these cells. Sonic hedgehog $(\mathrm{SHH})$ derived from astrocytes promotes the maturity and integrity of the BBB by upregulating tight junctions (TJs) in brain capillary endothelial cells (ECs). However, the effect of $\mathrm{SHH}$ on BBB in $\mathrm{ICH}$ has not been investigated.

Methods: Cyclopamine (CYC) is a potent, selective inhibitor that specifically blocks the $\mathrm{SHH}$ signaling pathway. Here, we used pharmacological inhibitions (CYC and its derivatives) to determine a critical role of the $\mathrm{SHH}$ signaling pathway in promoting BBB integrity after $\mathrm{ICH}$ by mechanisms of regulating the TJ proteins in vivo and in vitro.

Results: The expression of astrocytic $\mathrm{SHH}$ was upregulated in mouse brains after $\mathrm{ICH}$. Compared with the vehicle-treated group, inhibition of the $\mathrm{SHH}$ signaling pathway with $\mathrm{CYC}$ and its derivatives treatments aggravated neurological function deficits, brain edema, hematoma volume, and BBB impairment by downregulating TJs in ECs through the $\mathrm{SHH}-\mathrm{Gli}-1$ axis in vivo and in vitro.

Conclusions: $\mathrm{SHH}$ signaling pathway at the level of the BBB provides a barrierpromoting effect, suggesting that the $\mathrm{SHH}$ signaling pathway may function as a potential therapeutic target for restoring BBB function in $\mathrm{ICH}$.

\footnotetext{
Keywords: cyclopamine, tight junctions, sonic hedgehog signaling pathway, blood brain barrier, cerebral hemorrhage

Abbreviations: ICH, intracerebral hemorrhage; BBB, blood-brain barrier; SHH, sonic hedgehog; TJs, tight junctions; ECs,
endothelial cells; CYC, cyclopamine; Ptch-1, patched-1; SMO, smoothened; Gli-1, glioma-associated oncogene homolog-1;
HPBCD, hydroxypropyl- $\beta$-cyclodextrin; CMC-NA, carboxymethylcellulose sodium; PBS, phosphate-buffered saline; mNSS,
modified Neurological Severity Score; GFAP, glial fibrillary acidic protein; EB, Evans blue; GAPDH, glyceraldehyde
3-phosphate dehydrogenase; PHE, perihematomal.
} 


\section{INTRODUCTION}

Intracerebral hemorrhage $(\mathrm{ICH})$ is a devastating stroke subtype with a high risk of morbidity and mortality (Xi et al., 2013). It accounts for 10-15\% of all stroke but lacks effective treatments. The 1st-year survival rate is only 38\% (Dennis et al., 1993; Qureshi et al., 2001). Patients who survive typically have a series of neurologic deficits. Primary brain injury commonly occurs immediately within the first few hours after the ictus of $\mathrm{ICH}$, leading to the direct entry of blood components (thrombin, fibrin, and erythrocyte components) into the brain and forming a hematoma that provokes mechanical damage (Keep et al., 2014; Tschoe et al., 2020). Neurons and glia are affected by the injury and induce oxidative stress, inflammatory activation, neurotransmitter release, membrane depolarization, and mitochondrial dysfunction, contributing to secondary brain injury. Following the activation of coagulation and hemoglobin breakdown, thrombin and clot components were released and initiate the entire cascade of cellular and molecular changes of the brain, leading to the further destruction of brain tissue (Madangarli et al., 2019). After ICH, microglia were rapidly activated to respond to these stimuli by communicating with the immune system and secreting products that cause blood-brain barrier (BBB) destruction, vasogenic edema, neuronal and glial apoptosis (Qureshi et al., 2009; Tschoe et al., 2020). Astrocytes actively accumulate in the perihematomal (PHE) region after $\mathrm{ICH}$ onset, participating in modulating $\mathrm{BBB}$ function and interaction with microglia and neurotrophic support (SukumariRamesh et al., 2012; Tschoe et al., 2020). Additionally, the integrity of the structure and function of brain microvascular endothelial cells (BMECs) change with $\mathrm{ICH}$ progression, and leading to BBB disruption (Yu et al., 2015).

The BBB, which is composed of capillary endothelial cells (ECs), pericytes, and perivascular astrocytes, selectively restricts the blood-to-brain paracellular compound diffusion and regulates cerebral homeostasis and proper neuronal function. After $\mathrm{ICH}$, the increase in leukocyte infiltration into the brain and $\mathrm{BBB}$ permeability occurs via the changes of paracellular (dysfunction of the tight junction) and transcellular (transcytosis) reactions across the endothelium, the ICH-induced inflammatory response can also cause BBB dysfunction (Keep et al., 2014). Also, Breakdown of the BBB leads to the infiltration of blood components into the brain parenchyma, and the subsequent mechanical stress, the release of toxic components and immune response further aggravate $\mathrm{BBB}$ destruction and brain edema.

Astrocytes are important components of the $\mathrm{BBB}$, which not only support the structure of $\mathrm{BBB}$ but also regulate $\mathrm{BBB}$ integrity via secreting factors. Sonic Hedgehog $(\mathrm{SHH})$ is a secreted glycoprotein molecule that participates in various physiological processes, such as neurogenesis, anti-inflammation, antioxidation, and anti-apoptosis (He et al., 2017; Caradu et al., 2018; Chen et al., 2018). Recent studies have revealed that $\mathrm{SHH}$ produced by astrocytes maintains the tightness and decreases the solute permeability of BBB by upregulating tight junctions (TJs) in ECs (Janzer and Raff, 1987; Alvarez et al., 2011, 2013; Obermeier et al., 2013). BBB ECs express an SHH receptor called patched-1 (Ptch-1), which is a kind of transmembrane protein, suppressed by $\mathrm{SHH}$ binding. The G-protein coupled receptor Smoothened (SMO) is activated by the repression of Ptch-1, resulting in the activation of the transcription factor gliomaassociated oncogene homolog-1 (Gli-1) and its translocation to SHH-target genes. Gli-1 is an important regulator of the downstream target genes that control the expressions of TJs and BBB formation.

However, the role of the astrocytic SHH signaling pathway in $\mathrm{BBB}$ integrity after $\mathrm{ICH}$ remains unclear. In this study, we investigated the influence of the astrocytic SHH signaling pathway on $\mathrm{ICH}$ and its potential mechanism in vivo and in vitro.

\section{MATERIALS AND METHODS}

\section{Animals, Reagents, and Study Design}

All experiments were approved by the Animal Experiments Ethical Committee of Tianjin Neurological Institute and Jining Medical University. Adult male C57BL/6 mice (20-23 g; 7-8 weeks old) were used in this experiment. All mice were housed in an asepsis condition with a controlled temperature of $21^{\circ} \mathrm{C}$, a standardized light-dark cycle, and normal amounts of food and water. They were allowed to acclimate for a week before experiments. All operations were performed while the animals were under anesthesia. In our study, we noted no difference in physiologic variables of mice among the experimental groups, including body weight, temperature, blood pressure, and arterial blood gas.

Cyclopamine (CYC; Selleck, Houston, TX, USA) is a smallmolecule steroidal alkaloid that specifically inhibits the $\mathrm{SHH}$ signaling pathway by blocking SMO and interfering with $\mathrm{SHH}$ signal transduction in SHH-responsive cells (Cooper et al., 1998; Chen et al., 2002). In vivo, hydroxypropyl- $\beta$-cyclodextrin (HPBCD, Sigma-Aldrich, St. Louis, MO, USA) was dissolved in phosphate-buffered saline (PBS; 45\% wt/vol) and used to prepare conjugated CYC at $1 \mathrm{mg} / \mathrm{ml}$. Mice were intraperitoneally injected with CYC at a concentration of $10 \mathrm{mg} / \mathrm{kg}$ or with the same volume of the vehicle (45\% HPBCD in PBS; Palma et al., 2005; Alvarez et al., 2011; Zhang et al., 2017). In cell culture, $10 \mu \mathrm{M}$ CYC was used to inhibit the $\mathrm{SHH}$ signaling pathway (Li et al., 2012; Wang et al., 2014; Sun et al., 2016). LDE-225 (Sonidegib) and GDC-0449 (vismodegib) are two developed drugs targeting the $\mathrm{SHH}$ signaling pathway by inhibiting SMO, in a similar way to CYC. The two CYC derivatives are synthetic, highly potent clinical oral medicine approved by the US Food and Drug Administration (FDA) for basal-cell carcinoma (BCC; Irvine et al., 2016; Lauressergues et al., 2016; Rimkus et al., 2016). In vivo, carboxymethylcellulose sodium (CMC-NA, Selleck, Houston, TX, USA) was dissolved in double-distilled water $(0.5 \% \mathrm{wt} / \mathrm{vol})$ and used to prepare conjugated LDE-225 at $4 \mathrm{mg} / \mathrm{ml}$ and GDC-0449 at $2 \mathrm{mg} / \mathrm{ml}$ (Selleck, Houston, TX, USA). Mice were delivered by daily oral gavage with LDE-225 at a concentration of $20 \mathrm{mg} / \mathrm{kg}$ and GDC-0449 at a concentration of $10 \mathrm{mg} / \mathrm{kg}$ or with the same volume of the vehicle (0.5\% CMC-NA in double-distilled water; Pan et al., 2010; Yang et al., 2015; Cazet et al., 2018). 
In vivo, animals were randomly divided into experimental groups according to the designated treatments: Sham-operated mice group (SHAM), sham-operated mice treated with $\mathrm{CYC} /$ LDE-225/ GDC-0449 group (SHAM+CYC/SHAM+LDE225/SHAM+GDC-0449), ICH-operated mice treated with the vehicle group (ICH+Vehicle), $\mathrm{ICH}$-operated mice treated with CYC/ LDE-225/ GDC-0449 group (ICH+CYC/ICH+LDE225/ICH+GDC-0449). In groups treated with vehicle and CYC, mice received a daily injection of an equal volume of vehicle or CYC for three consecutive days starting from the day of operation (Figure 2A). In groups treated with vehicle and LDE-225/ GDC-0449, mice received daily oral gavage of an equal volume of vehicle or LDE-225/ GDC-0449 for three consecutive days starting from the day of operation (Figures 3A,E). In vitro, cells were assigned into four groups: ECs cultured alone (bEnd3), ECs co-cultured with sham-astrocytes (SHAM), ECs co-cultured with ICH-astrocytes and vehicle (ICH+Vehicle), ECs co-cultured with $\mathrm{ICH}$-astrocytes and $\mathrm{CYC}(\mathrm{ICH}+\mathrm{CYC})$.

\section{Establishment of Mouse ICH Model}

In this study, to confirm our findings in ICH, an autologous blood-induced ICH model was also involved to exclude the potential influence of $\mathrm{CYC}$ on collagenase. Injection of collagenase or autologous blood was used to induce ICH in mice as previously described (Grossetete and Rosenberg, 2008; Rynkowski et al., 2008; Li et al., 2017a,c). A mixture of ketamine $(100 \mathrm{mg} / \mathrm{kg})$ and xylazine $(10 \mathrm{mg} / \mathrm{kg})$ was used to anesthetize mice by intraperitoneal injection. On the right side of the mouse skull, a $1 \mathrm{~mm}$ burr hole was drilled (stereotaxic coordinates: $0.5 \mathrm{~mm}$ anterior to the Bregma and $2.3 \mathrm{~mm}$ lateral from the midline). The collagenase injection model was induced: $0.0375 \mathrm{U}$ bacterial collagenase (Type IV; Solarbio, Beijing, China) in 0.5 $\mu \mathrm{l}$ saline was injected at a rate of $1 \mu \mathrm{l} / \mathrm{min}$ into the right caudate nucleus $(3.7 \mathrm{~mm}$ below the skull surface) using a $10 \mu \mathrm{l}$ syringe driven by an infusion pump (KD Scientific, Holliston, MA, USA). The autologous blood injection model was induced $30 \mu \mathrm{l}$ autologous blood was obtained from the angular vein of the mouse and then injected using a $50 \mu l$ syringe driven by an infusion pump (KD Scientific, Holliston, MA, USA) as previously described (Li et al., 2017c). The first $5 \mu \mathrm{l}$ autologous blood was infused at a depth of $3 \mathrm{~mm}$ with a rate of $1 \mu \mathrm{l} / \mathrm{min}$ to generate a clot under the hole. The needle was then placed to a depth of $3.7 \mathrm{~mm}$ and paused for $5 \mathrm{~min}$. After the pause, the remaining 25 $\mu l$ of autologous blood was infused at the same rate. Then the needle was slowly withdrawn at $1 \mathrm{~mm}$ increments after pausing for $20 \mathrm{~min}$, each for three movements at the 5-min interval. In the sham group, mice were injected with the same dose of saline. Ultimately, the skull hole was sealed with sterile bone wax and the skin incision was sterilized and sutured using standard techniques. Then animals were placed in standard mouse cages with a free supply of water and food.

\section{Neurological Behavioral Assessment}

Neurological behavioral assessment including the modified Neurological Severity Score (mNSS; Jin et al., 2017; Li et al., 2017) and the Rotarod test (Moriguchi et al., 2012; Yang et al., 2018) was performed on days 1 and 3 after sham and ICH surgery by two observers who were blinded to the experiments.

The mNSS test is a composite measurement to comprehensively assess the motor (test of raising mouse by the tail and placing the mouse on the floor), sensory (visual test, tactile test, and proprioceptive test), reflex (reflexes absent and abnormal movements) and balance (beam balance tests) functions (Supplementary Table 1; Li et al., 2017b). The scale of the mNSS is from 0 to 18 . A score of $13-18$ indicates severe impairment, 7-12 indicates moderate impairment, and 1-6 indicates mild impairment (Ren et al., 2018). One point was given if mice failed to complete a task or lacked a tested reflex.

The rotarod test measures the systemic motor abilities, specifically the balance and coordination of the ICH mice (Yang et al., 2018). Mice practiced running on an automated accelerating rotarod treadmill $(3 \mathrm{~cm}$ in diameter and $30 \mathrm{~cm}$ length, with a non-slippery surface $20 \mathrm{~cm}$ above the base). The rod contained five equal sections, which were divided by four disks, enabling five mice to walk on the rod at the same time. Test sessions consisted of three trials, in which the fixed acceleration mode's rotational speed accelerated from 0 to $40 \mathrm{rpm}$. Each trial lasted for $5 \mathrm{~min}$ with a 30 -min interval between two trials. The final score was recorded as the average time over three trials while mice were able to remain on the rotarod. Mice were positioned on the rotating rod drum up to $5 \mathrm{~min}$. Before $\mathrm{ICH}$, mice were tested on the rotating rod $(4 \mathrm{rpm})$ over 3 days, and only those who stayed on the rod for at least $60 \mathrm{~s}$ were subjected to ICH.

\section{Measurement of Brain Water Content}

Brain water content was assessed at day 3 after sham and $\mathrm{ICH}$ surgery (Han et al., 2017). Mice brains were harvested and separated into three parts without perfusion: the left cerebral hemisphere, the right cerebral hemisphere, and the cerebellum. The samples were quickly weighed on a precise electronic scale (wet weight) and then dried at $100^{\circ} \mathrm{C}$ for $24 \mathrm{~h}$ to measure the dry weight. The following formula was used to calculate the percentage of edema in the mouse brain: (wet weight - dry weight)/wet weight $\times 100 \%$.

\section{Assessment of Hemorrhage Volume}

On day 3 after sham and ICH surgery, mouse brains were collected and post-fixed in $4 \%$ paraformaldehyde overnight at $4^{\circ} \mathrm{C}$. In slices of $1 \mathrm{~mm}$ thickness, hemorrhage sizes were gaged. After photographing these slices, hematoma volumes were measured using ImageJ software (NIH, Bethesda, MD, USA, 1997-20091).

\section{Immunofluorescent Staining and Analysis}

On day 3 after sham and ICH surgery, mouse brains were collected and post-fixed in $4 \%$ paraformaldehyde overnight at $4^{\circ} \mathrm{C}$. The fixed brains were then removed and dehydrated using $15 \%$ and $30 \%$ sucrose in PBS. Subsequently, brain sections $(8 \mu \mathrm{m})$ were prepared using a Leica Microsystems LM3050S cryostat (Leica Microsystems, Wetzlar, Hessen, Germany). Brain

\footnotetext{
${ }^{1}$ http://rsb.info.nih.gov/ij/
} 

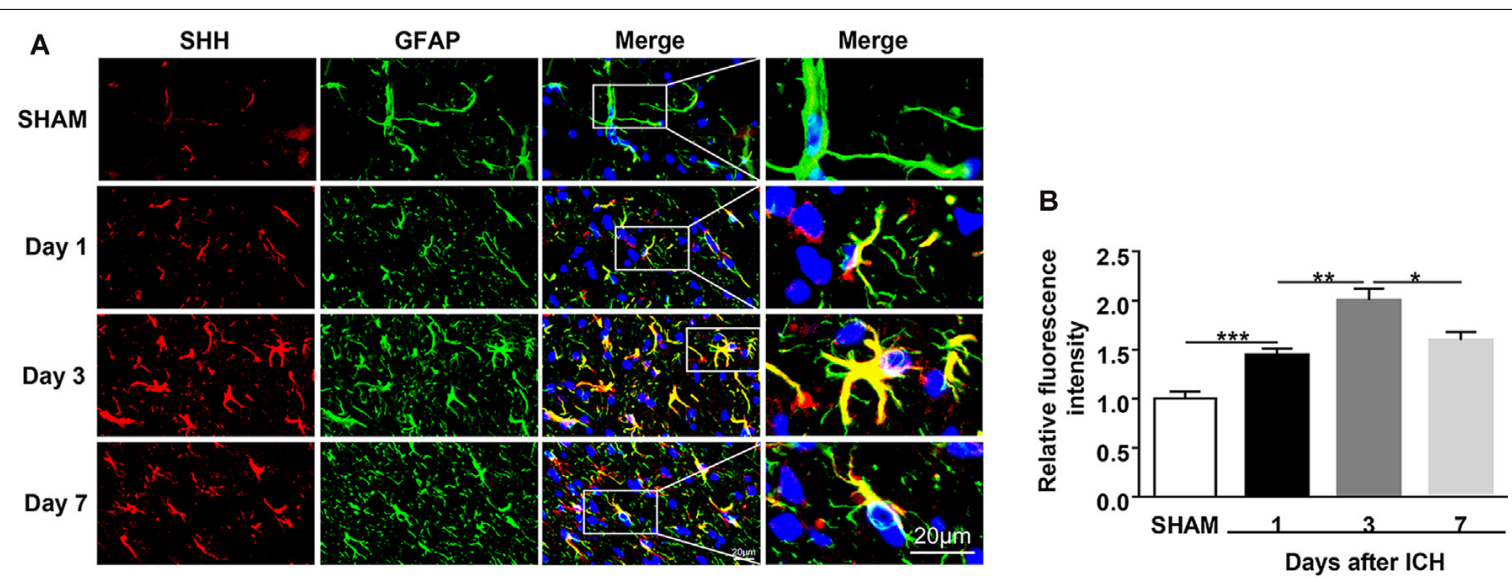

C

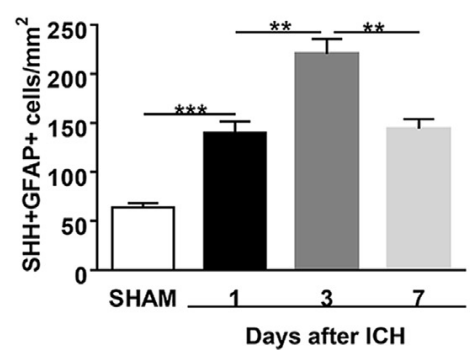

D

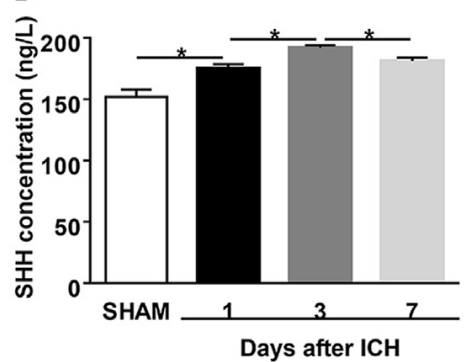

FIGURE 1 | Upregulation of sonic hedgehog $(\mathrm{SHH})$ expression in the astrocytes of intracerebral hemorrhage (ICH) mice. ICH was induced in mice by injection of autologous blood. (A,B) Representative images and quantification of fluorescence intensity showed SHH (red) in astrocytes (glial fibrillary acidic protein, GFAP, green) in mouse perihematomal (PHE) brain tissues at days 1, 3, and 7 after sham or ICH surgery, $n=6$ mice per group. (C) Quantification of the SHH ${ }^{+}$GFAP ${ }^{+}$cells in mouse PHE brain tissues at days 1, 3, and 7 after surgery, $n=6$ mice per group. (D) The protein levels of SHH in mouse PHE brain tissues at days 1,3 , and 7 after sham or $\mathrm{ICH}$ surgery were measured by ELISA kits, $n=3$ mice per group. Data are presented as mean $\pm \mathrm{SEM}$. ${ }^{\star} P<0.05,{ }^{\star \star} P<0.01,{ }^{\star \star \star} P<0.001$.

tissues were permeabilized with $0.3 \%$ Triton X-100 for $10 \mathrm{~min}$. After blocking with $3 \%$ bovine serum albumin for $1 \mathrm{~h}$ at ambient temperature, slides were incubated with primary antibodies: rat anti-SHH (25 $\mu \mathrm{g} / \mathrm{ml}$; Abcam, Cambridge, MA, USA), goat anti-glial fibrillary acidic protein (GFAP; 1:500; Abcam, Cambridge, MA, USA), rabbit anti-Ptch-1 (1:500; Affinity Biosciences, Cincinnati, OH, USA), mouse anti-SMO (1:100; Santa Cruz Biotechnology, Santa Cruz, CA, USA) and mouse anti-Gli-1 (1:100; Santa Cruz Biotechnology, Santa Cruz, CA, USA) overnight at $4^{\circ} \mathrm{C}$. For in vitro staining, cells were inoculated on coverslips, fixed, and incubated with the following primary antibodies: rabbit anti-claudin-5 (1:100; Abcam, Cambridge, MA, USA) and rabbit anti-ZO-1 (1:100; Abcam, Cambridge, MA, USA). The next day, cells were washed with PBS $(5 \times 5 \mathrm{~min})$ and then incubated at ambient temperature for $1 \mathrm{~h}$ with designated secondary antibodies (1:1,000; all from Invitrogen, Carlsbad, CA, USA): rabbit anti-rat 594, rabbit anti-goat 488, goat anti-mouse 488, donkey anti-rabbit 488 . Finally, tissues were counterstained for cell nuclei with $4^{\prime}, 6$ diamidino-2-phenylindole. The intensity of the staining was measured semi-quantitatively using ImageJ software. The cell numbers were expressed as the $\mathrm{SHH}+\mathrm{GFAP}+$ cell numbers per square millimeter.

\section{ELISA}

We separately collected samples of mice brain at 1, 3, and 7 days after ICH and the co-cultured supernatants for $24 \mathrm{~h}$ to determine the contents of SHH and MMP-9 by ELISA assay (SenBeiJia, Nanjing, China), according to the manufacturer's protocol under uniform conditions. Determinations were performed in duplicate on the individual sample. Results expressed from each sample were normalized for protein concentration.

\section{Measurement of Evans Blue}

The $\mathrm{BBB}$ integrity was evaluated using 2\% Evans Blue dye (EB; $2 \mathrm{ml} / \mathrm{kg}$; Solarbio, Beijing, China), which was injected into the tail vein $2 \mathrm{~h}$ before sacrifice at day 3 after sham and ICH surgery. Mouse brain tissues were harvested and the ipsilateral hemisphere was weighed on an electronic balance. After homogenization with formamide in a test tube, the ipsilateral hemisphere was incubated at $60^{\circ} \mathrm{C}$ for $72 \mathrm{~h}$. After centrifuged at $1,000 \times \mathrm{rpm}$ for $5 \mathrm{~min}$, the supernatants were collected and added into a microplate along with standards. The optical density of the extracted dye in each sample was assessed using a microplate reader (Thermo Scientific, San Diego, CA, USA; $\lambda=450,570 \mathrm{~nm}$ ). The following formula was used to 


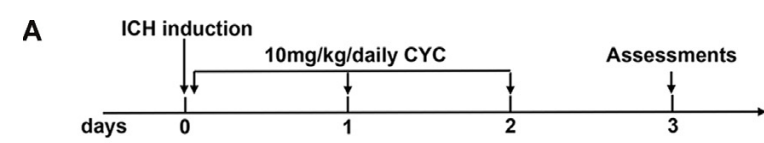

B
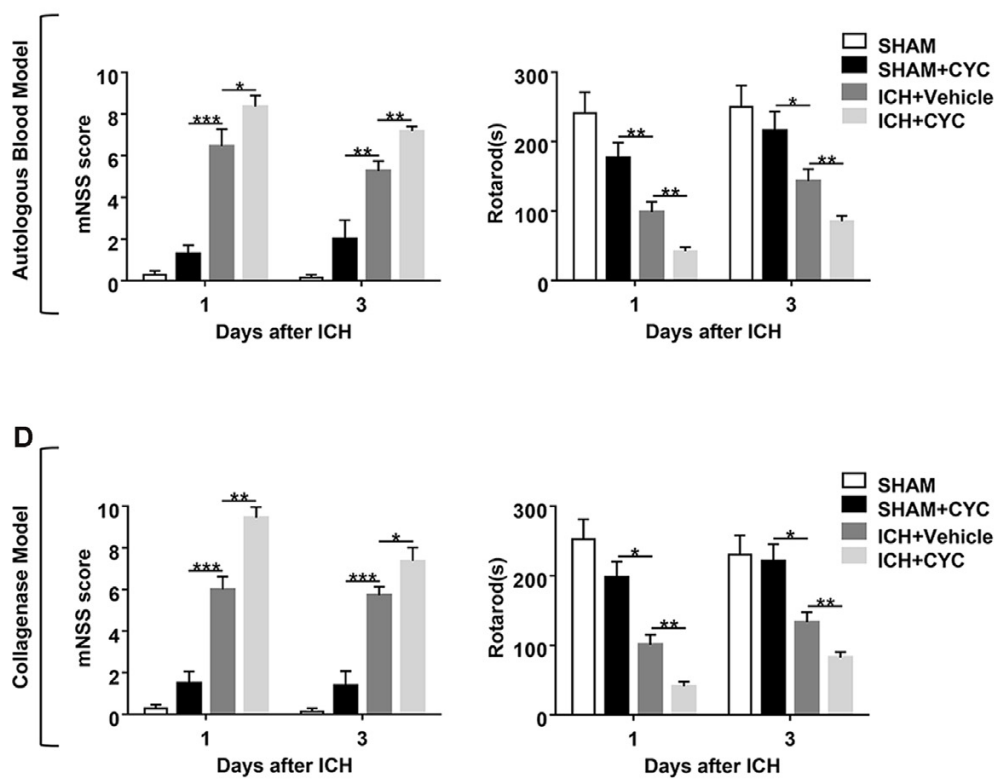

C

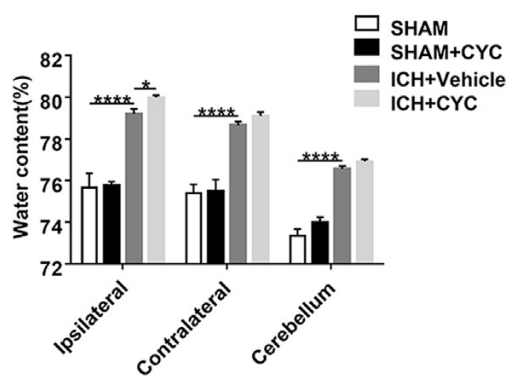

E

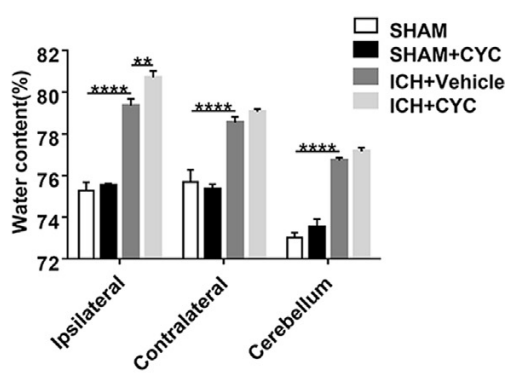

$\mathbf{F}$

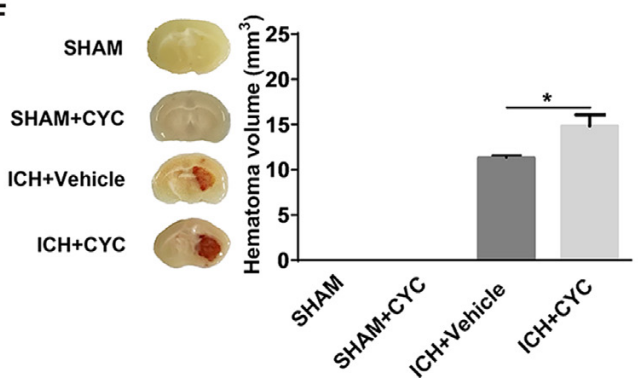

FIGURE 2 | Blockage of the SHH signaling pathway by cyclopamine (CYC) aggravates ICH-induced brain injury in mice. (A) The schematic diagram illustrates the CYC treatment and study design. Mice were treated with CYC (10 mg/kg) or an equal volume of vehicle by intraperitoneal injection once a day, starting from day 0 to day 2 after sham or ICH operation. (B,D) The neurological function assessments of modified Neurological Severity Score (mNSS) and rotarod tests were performed to evaluate the motor, sensory, reflex, and balance functions in mice treated with CYC or vehicle on day 1 and day 3 after injection of saline, autologous blood (B), or collagenase (D), $n=6-11$ mice per group. (C,E) Measurement of brain water content in mice treated with CYC or vehicle at day 3 after injection of saline, autologous blood (C), or collagenase (E), $n=3-8$ mice per group. (F) Measurement of hematoma volume in mice treated with $\mathrm{CYC}$ or vehicle at day 3 after injection of saline or collagenase, $n=3$ mice per group. Data are presented as mean \pm SEM. ${ }^{\star} P<0.05,{ }^{\star \star} P<0.01,{ }^{\star \star \star} P<0.001,{ }^{\star \star \star \star} P<0.0001$.

calculate the concentration of EB by linear regression: EB content in brain tissue $(\mu \mathrm{g} / \mathrm{g}$ wet brain $)=\mathrm{EB}$ concentration $\times$ formamide $(\mathrm{ml})$ /wet weight $(\mathrm{g})$.

\section{Western Blot Analysis}

On day 3 after sham and ICH surgery, the fresh ipsilateral hemispheres of mouse brains samples were homogenized by sonication in RIPA buffer (Solarbio, Beijing, China) containing protease complete inhibitor cocktail. The supernatants were collected after centrifugation. In vitro, after co-cultured for $24 \mathrm{~h}$, cells were harvested into RIPA buffer containing cocktail. The Protein concentration was assessed by using the BCA protein assay kit (Solarbio, Beijing, China). Protein samples were separated on $10 \%$ SDS-PAGE gels and then transferred onto PVDF membranes (Millipore, Billerica, CA, USA), which were blocked with $5 \%$ non-fat milk for $1 \mathrm{~h}$ at ambient temperature. The membranes were incubated at $4^{\circ} \mathrm{C}$ overnight with the following primary antibodies: rabbit anti-ZO-1 (1:1,000; Millipore, Billerica, CA, USA), rabbit anti-Occludin (1:1,000; Millipore, Billerica, CA, USA), rabbit anti-Claudin-5 (1:1,000; Millipore, Billerica, CA, USA), mouse anti-SMO (1:1,000; Santa Cruz Biotechnology, Santa Cruz, CA, 


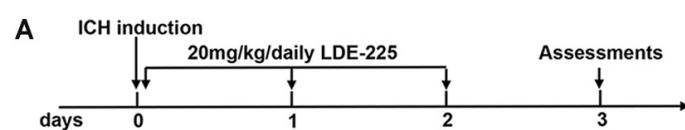

B
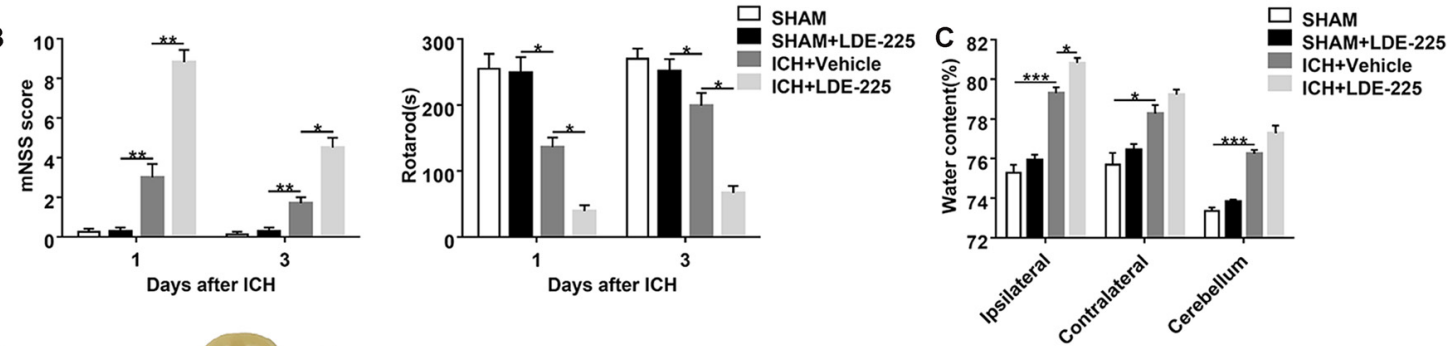

D

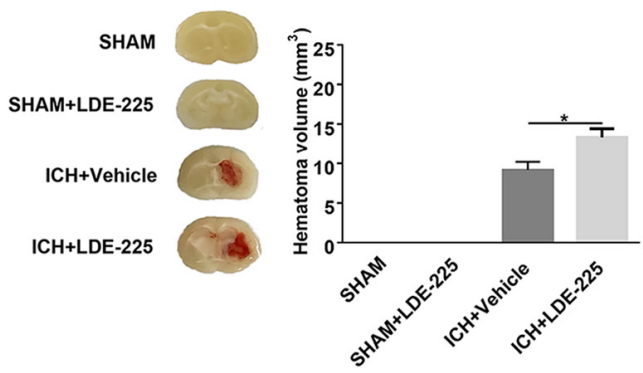

E
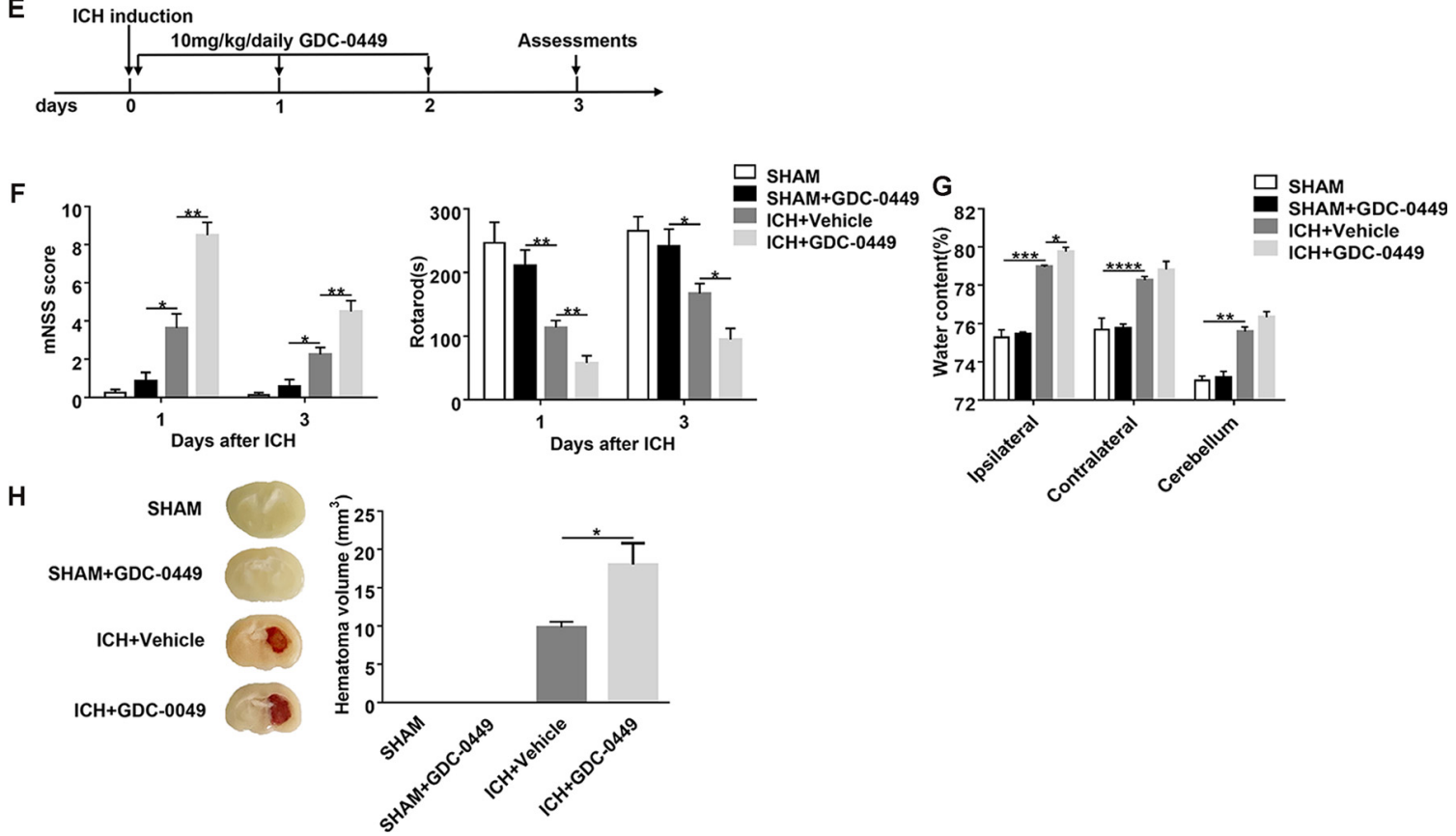

FIGURE 3 | Blockage of SHH signaling pathway by CYC derivatives (LDE-225 and GDC-0449) aggravates ICH-induced brain injury in mice. (A,E) The schematic diagram illustrates LDE-225 or GDC-0449 treatment and study design. Mice received daily oral gavage of LDE-225 (20 mg/kg) or GDC-0449 (10 mg/kg), or with an equal volume of vehicle, starting from day 0 to day 2 immediately after sham or ICH operation. (B,F) The neurological function assessments of mNSS and rotarod tests were performed to evaluate the motor, sensory, reflex, and balance functions in mice treated with LDE-225/GDC-0449 or vehicle on day 1 and day 3 after injection of saline or collagenase, $n=6-10$ mice per group. (C,G) Measurement of brain water content in mice treated with LDE-225/GDC-0449 or vehicle on day 3 after injection of saline or collagenase, $n=3$ mice per group. (D,H) Measurement of hematoma volume in mice treated with LDE-225/GDC-0449 or vehicle on day 3 after injection of saline or collagenase, $n=3$ mice per group. Data are presented as mean \pm SEM. ${ }^{\star} P<0.05,{ }^{\star \star} P<0.01,{ }^{\star \star \star} P<0.001,{ }^{\star \star \star \star} P<0.0001$.

USA), mouse anti-Gli-1 (1:1,000; Santa Cruz Biotechnology, Santa Cruz, CA, USA) and mouse anti-glyceraldehyde 3phosphate dehydrogenase (GAPDH; 1:1,000; ZSGB-BIO,
Beijing, China). The next day, the membranes were washed and incubated with a species-appropriate secondary antibody for $1 \mathrm{~h}$ at ambient temperature. After three washes, the intensity of the 
protein band was examined by densitometry (Bio-Rad, Hercules, CA, USA) which was analyzed using ImageJ software.

\section{Isolation of Primary Astrocytes}

Primary astrocytes were sorted from mouse brain tissues at day 3 after sham and $\mathrm{ICH}$ surgery as previously described (Li et al., 2018; Scheuer et al., 2019). Briefly, brains were gently removed, chopped with scissors in ice-cold Dulbecco's Modified Eagle's Medium, and then digested with papain (Sigma-Aldrich, St. Louis, MO, USA) at $37^{\circ} \mathrm{C}$ for $30 \mathrm{~min}$. Then cell suspensions were fractionated by $30 \%$ Percoll gradients at $700 \times \mathrm{g}$ for $10 \mathrm{~min}$ to get rid of myelin. After resuspended in $1 \%$ fetal bovine serum, cell suspensions were filtered with a cell strainer to obtain mononuclear cells. For magnetic labeling of astrocytes, $1 \times 10^{6}$ cells per $60 \mu 11 \%$ fetal bovine serum were incubated with anti-Glast-1 antibody, which is marked with PE (Novus, Saint Charles, Missouri, MO, USA) for $30 \mathrm{~min}$ at $4^{\circ} \mathrm{C}$. After washed with buffer, $1 \times 10^{6}$ cells per $20 \mu$ l buffer were incubated with anti-PE microbeads (Miltenyi Biotec, Bergisch Gladbach, NRW, Germany) for $20 \mathrm{~min}$ at $4^{\circ} \mathrm{C}$. Magnetically labeled astrocytes were then isolated after several washes with $2 \mathrm{ml}$ buffer. The purity of the primary astrocytes was determined by flow cytometry (BD Biosciences, San Jose, CA, USA) before culture (>98\%).

\section{Cell Culture and Treatment}

A mouse brain endothelial cell line, bEnd3 (Zqxzbio, Shanghai, China), was used in this study. bEnd3 cells were seeded into 24-well plates with a density of $1 \times 10^{5}$ cells per well and grown in high-glucose Dulbecco's Modified Eagle's Medium. After culturing the bEnd 3 cells in a $5 \% \mathrm{CO}_{2} / 95 \%$ air incubator at $37^{\circ} \mathrm{C}$ for 3 days, primary astrocytes were seeded into the plate with a density of $1 \times 10^{5}$ cells per well and co-cultured with the bEend 3 cells for $24 \mathrm{~h}$. Then, co-cultured cells were incubated with $\mathrm{CYC}$ or an equal volume of the vehicle for $24 \mathrm{~h}$ before harvesting for subsequent treatments.

\section{Statistical Analysis}

Data were shown as mean \pm SEM and analyzed using GraphPad Prism 6.0 software (GraphPad Software, La Jolla, CA, USA). Two-tailed unpaired student's $t$-test was used to determine the differences between the two groups. One-way ANOVA (KruskalWallis test) followed by the Tukey post hoc test or 2-way ANOVA followed by the Bonferroni post hoc test was used for multigroup comparison. $P<0.05$ was considered statistically significant.

\section{RESULTS}

\section{Upregulation of SHH Expression in Astrocytes of the Perihematomal (PHE) Brain Tissues After ICH}

To identify the brain expression of $\mathrm{SHH}$ after $\mathrm{ICH}$, we injected mice with autologous blood and measured the expression of $\mathrm{SHH}$ in the brain using immunostaining and ELISA at days 1, 3 , and 7 after $\mathrm{ICH}$. We found that $\mathrm{SHH}$ was mainly expressed in astrocytes and its expression was significantly increased on the first day after ICH as compared to the sham group, the protein level of SHH was persistently increased on day 3, but downregulated at day 7 after ICH (Figures 1A-D). The dynamic expression of $\mathrm{SHH}$ in the brain indicates the participation of astrocytic $\mathrm{SHH}$ in the pathogenesis after $\mathrm{ICH}$.

\section{Inhibition of SHH Signaling Pathway Aggravates Neurological Deficits and Brain Injury in Mice After ICH}

To assess the effect of the $\mathrm{SHH}$ signaling pathway on $\mathrm{ICH}$, we used SMO inhibitors (CYC, LDE-225, and GDC0449), antagonists of SHH downstream key molecule SMO, to selectively inhibit the activation of the $\mathrm{SHH}$ signaling pathway. There was no significant change in neurological deficits and brain injury in SMO inhibitors-treated mice. After ICH surgery, neurological deficits and brain injury were aggravated. We found that mice treated with SMO inhibitors showed severer neurological function deficits compared with the ones receiving vehicle at day 1 and day 3 after ICH surgery (Figures 2B,D, $3 \mathrm{~B}, \mathrm{~F})$. Compared with the vehicle-treated group, the brain water contents in mice injected with SMO inhibitors were significantly increased at day 3 after ICH surgery (Figures 2C,E, 3C,G). Compared with the vehicle-treated group, the hematoma sizes in mice injected with SMO inhibitors were significantly increased at day 3 after ICH surgery (Figures 2F, 3D,H). Meanwhile, hematoma sizes showed to be correlated with the behavioral scores in CYC-treated mice. Severer neurological deficits tend to be found in mice with larger hematoma sizes (Supplementary Figure 2). These data suggest that the inhibition of the $\mathrm{SHH}$ signaling pathway exacerbates ICH-induced brain injury.

\section{Blockage of SHH Signaling Pathway Exacerbates BBB Damage in Mice After ICH}

To identify the role of SMO inhibitors in BBB dysfunction after ICH, EB extravasation, and the expressions of TJs were examined. Compared with the sham group, there was no significant change of EB extravasation in SMO inhibitorstreated mice. After ICH surgery, EB extravasation was increased. Compared with the vehicle-treated group, SMO inhibitors treatments increased the leakage of $\mathrm{BBB}$ at day 3 after $\mathrm{ICH}$ (Figures 4A-F). Western blot analysis of TJs also showed the decrease of brain expressions of ZO-1, occludin, and claudin-5 in $\mathrm{ICH}+\mathrm{CYC}$ groups at day 3 after ICH surgery as compared to the vehicle-treated controls (Figures 4G,H). These data indicate that the $\mathrm{SHH}$ signaling pathway has a critical effect on BBB integrity after ICH.

\section{Inhibition of SHH Signaling Pathway Downregulates the Expressions of Tight Junctions in Endothelial Cells After ICH in vitro}

To further explore whether the $\mathrm{SHH}$ signaling pathway is involved in the regulation of TJs after $\mathrm{ICH}$, we co-cultured astrocytes with brain ECs. Astrocytes were sorted from the brain tissues of sham and ICH mice on day 3 after surgery. They were co-cultured with bEend3 cells for $24 \mathrm{~h}$ (Figures 5A,B) followed by the treatment with CYC or 
A

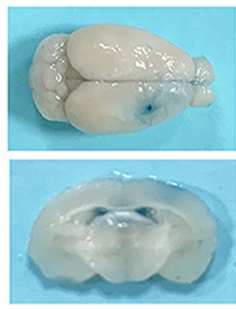

SHAM

C

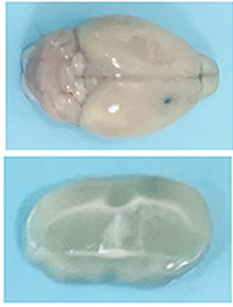

SHAM

E

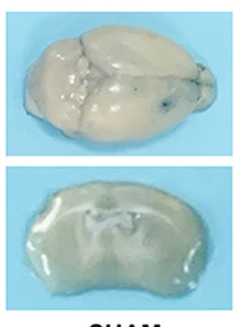

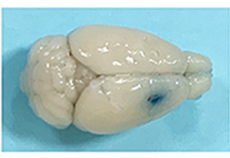

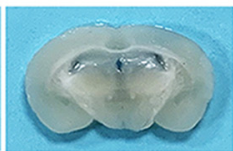

SHAM+CYC

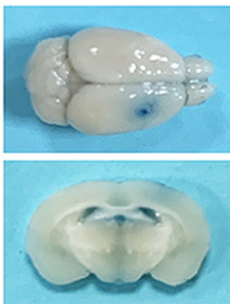

SHAM+LDE-225

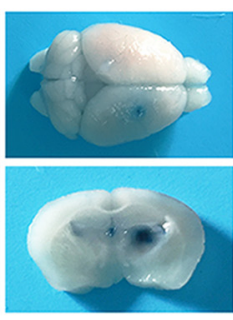

$\mathrm{ICH}+$ Vehicle

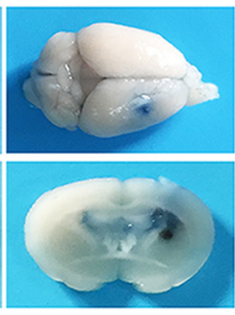

$\mathrm{ICH}+$ Vehicle

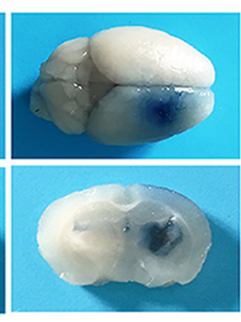

$\mathrm{ICH}+\mathrm{CYC}$

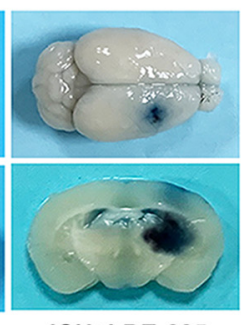

ICH+LDE-225
SHAM
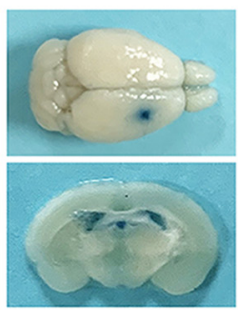

SHAM+GDC-0449
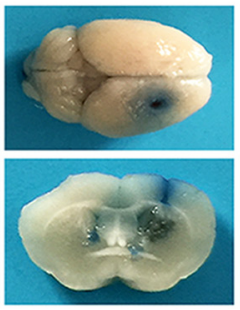

$\mathrm{ICH}+$ Vehicle

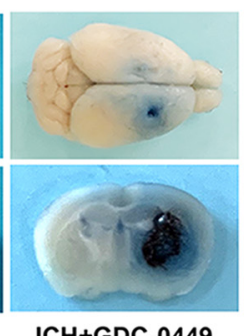

ICH+GDC-0449

B
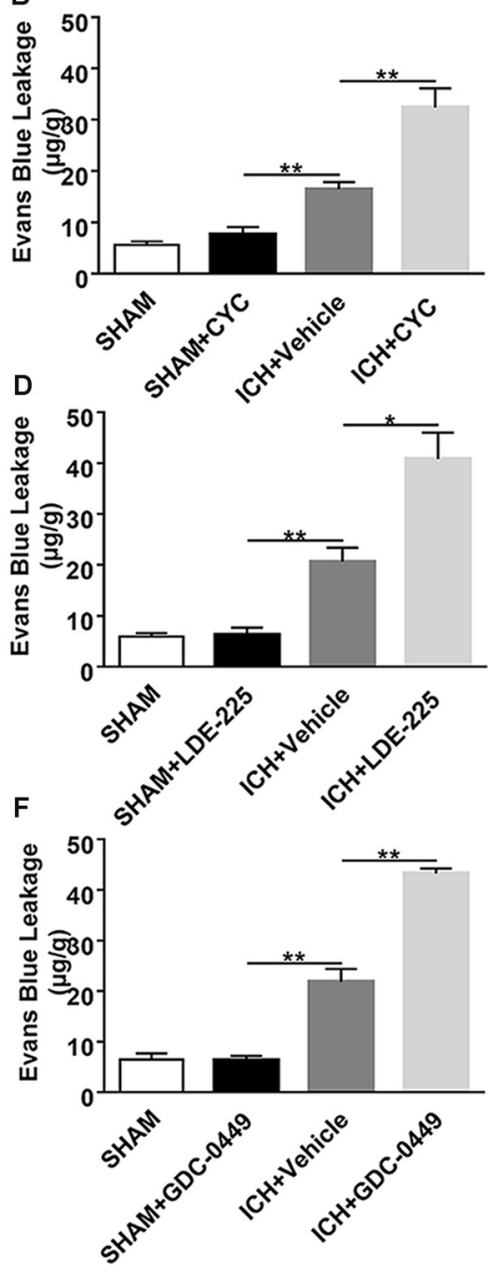

G

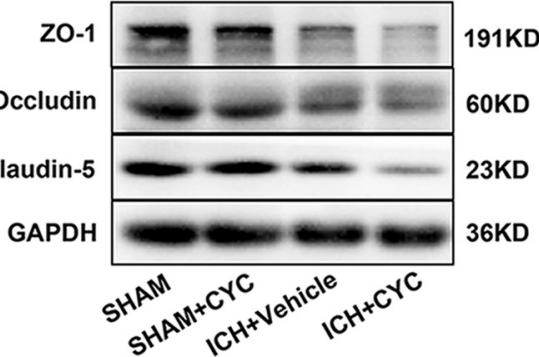

$191 \mathrm{KD}$

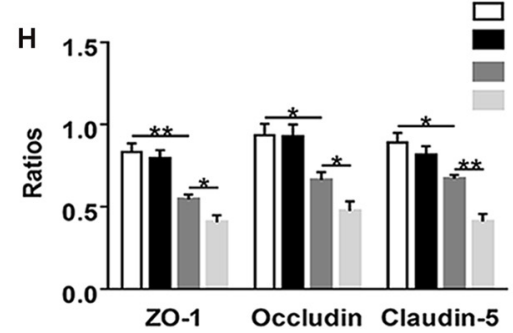

SHAM

SHAM+CYC

ICH+Vehicle $\mathrm{ICH}+\mathrm{CYC}$

FIGURE 4 | Inhibition of the SHH signaling pathway aggravates blood-brain barrier (BBB) permeability in mice after ICH. ICH was induced in mice by injection of collagenase. Mice received intraperitoneal injections of CYC (10 mg/kg) or an equal volume of the vehicle once a day, starting from day 0 to day 2 immediately after sham or ICH operation. Mice received daily oral gavage of LDE-225 (20 mg/kg) or GDC-0449 (10 mg/kg), or with an equal volume of vehicle, starting from day 0 to day 2 immediately after sham or ICH operation. The BBB integrity of mouse brain tissues was assessed at day 3 after sham or ICH surgery. (A-F) Histology images showed the Evans Blue (EB) dye leakage in the brain tissues of mice treated with SMO inhibitors or vehicle, $n=3-7$ mice per group. (G,H) Western blot images showed the expressions of tight junctions (TJs) in the brain tissues of mice treated with CYC or vehicle, $n=4-8$ mice per group. Data are presented as mean \pm SEM. ${ }^{*} P<0.05,{ }^{* *} P<0.01$.

vehicle. To explore the effects of astrocytic SHH on TJs, we measured the expressions of $\mathrm{ZO}-1$ and claudin- 5 in bEend 3 cells. There were no obvious differences between ZO-1 and claudin-5 expressions between the bEnd3 group and the SHAM group. Compared with the two groups above, the $\mathrm{ICH}+$ Vehicle group showed a significant reduction in the expressions of ZO-1 and claudin-5 in bEnd3 cells. Furthermore, the $\mathrm{ICH}+\mathrm{CYC}$ group showed a robust reduction in the expressions of ZO- 1 and claudin-5 in bEnd 3 cells as compared with the vehicle-treated group (Figures $\mathbf{5 C}-\mathbf{H}$ ). These 
data clarify the vital role of the astrocytic $\mathrm{SHH}$ signaling pathway in promoting $\mathrm{BBB}$ integrity via the regulation of TJs expressions.

\section{Blockage of SHH Signaling Pathway Suppresses ICH-Induced Activation of SHH-Gli-1 Axis in ICH Mice}

Based on the above results, we performed further experiments to identify the downstream mechanism under CYC inhibition of the SHH signaling pathway. The expressions of SHH-Gli-1 axis-related proteins were examined using immunofluorescent staining. Results showed that the expressions of Ptch-1, SMO, and Gli-1 in ICH+Vehicle groups were increased as compared to the SHAM group, indicating the $\mathrm{SHH}$ signaling pathway activation induced by ICH. The expressions of Ptch-1, SMO, and Gli-1 in $\mathrm{ICH}+\mathrm{CYC}$ groups were decreased compared to $\mathrm{ICH}+\mathrm{Vehicle}$ groups on day 3 after ICH surgery (Figures 6A-F). To further elucidate the SHH pathways under CYC inhibition, changes of SMO and Gli-1 were also identified in vitro (Supplementary Figure 3). These findings suggest that the inhibition of the SHH signaling pathway by CYC treatment suppresses the activation of the SHH-Gli-1 axis in mice with $\mathrm{ICH}$.

\section{DISCUSSION}

This study provides novel evidence that astrocytic $\mathrm{SHH}$ plays a protective role in $\mathrm{BBB}$ integrity after $\mathrm{ICH}$. We demonstrated that the level of astrocytic $\mathrm{SHH}$ was significantly elevated in $\mathrm{PHE}$ brain tissue of the animal model in the early stage of ICH, and astrocytes were the primary cell type that expressed $\mathrm{SHH}$. The inhibition of the SHH signaling pathway using SMO inhibitors treatment aggravated neurological impairments, brain edema, hematoma volume, and BBB permeability in the early stage of $\mathrm{ICH}$ in mice. Furthermore, the in vivo and in vitro data proved that astrocytic SHH improved the expressions of TJs in ECs through the SHH-Gli-1 axis and thus played a protective role in $\mathrm{BBB}$ integrity (Figure 7). Taken together, these results indicate that astrocytic SHH may be a potential therapeutic target for the management of ICH injury.

Astrocytes are essential for the maintenance of BBB structural integrity. They connect to the CNS vasculature and their end-feet sheathe the brain microvessels (Sukumari-Ramesh et al., 2012). Several studies suggested that a range of astrocyte-derived factors are responsible for BBB repair after acute brain injury (Michinaga and Koyama, 2019). After ICH, activated astrocytes are involved in the development of cytotoxic edema via expressing aquaporin4 , a water channel protein released from the end-feet of astrocytes (Laird et al., 2010). The role of astrocytes is known to be crucial to the SHH signaling pathway as astrocytes are considered a primary cellular source of $\mathrm{SHH}$ production in CNS injury (Amankulor et al., 2009; Alvarez et al., 2011; He et al., 2013; Bohannon et al., 2019). The upregulation of SHH in astrocytes was reported in TBI, EAE, and LPS injury models (Wang et al., 2008; Amankulor et al., 2009). One recent research demonstrated that CNS injuries induce a high $\mathrm{SHH}$ expression in mice brains, and astrocytes can act like stem cells with neurosphere formation in vitro (Sirko et al., 2013). Here, we firstly clarified the protective effect of astrocyte-derived $\mathrm{SHH}$ on $\mathrm{BBB}$ integrity after $\mathrm{ICH}$. These data supported the notion that astrocytes contributed to the formation and progression of $\mathrm{ICH}$-induced brain edema.

Many previous studies have used co-culture with primary astrocytes or astrocyte-conditioned media to enhances $\mathrm{BBB}$ characteristics and formation of TJs in brain ECs (Arthur et al., 1987; Isobe et al., 1996; Gaillard et al., 2001). However, some evidence indicates that co-culture with astrocytes or astrocyte-conditioned media didn't change the expressions of TJs in brain ECs (Brown et al., 2007; Li et al., 2010). The possible reasons for the opposite results may attribute to the variances in the culture models, culture conditions, and cell lines. Thus, the factors produced in the co-cultured environment may affect the BBB permeability. Astrocyte-derived endothelins (ETs), nitric oxide (NO), and vascular endothelial growth factors (VEGFs) cause reduction of TJs and endothelial apoptosis in $\mathrm{BBB}$ dysfunction, on the contrary, apolipoprotein E (APOE), insulin-like grow factor-1 (IGF-1), glial-derived neurotrophic factor (GDNF) and retinoic acid (RA) cause increase of TJs and protect ECs, resulting in BBB repair (Michinaga and Koyama, 2019). Astrocytes play a crucial role in pathogenic progression at the acute stages of ICH (Chiu et al., 2017). Astrocytes activity and astrocyte-derived factors are responsible for both $\mathrm{BBB}$ destruction and repairment after brain injury (Michinaga and Koyama, 2019). Following ICH, astrocyte-derived matrix metalloproteinases (MMPs) have been reported to aggravate $\mathrm{BBB}$ breakdown and brain damage with possible regulation on TJs (Min et al., 2015; Keep et al., 2018). We found that compared with the bEnd3 monoculture group, co-culture with sham-astrocytes didn't alter the expressions of ZO- 1 and claudin-5 in bEnd 3 cells, whereas co-culture with $\mathrm{ICH}$-induced astrocytes and vehicle led to a significant decrease in the expressions of ZO- 1 and claudin- 5 in bEnd3 cells. To investigate the potential factors that may alter the $\mathrm{BBB}$ permeability following $\mathrm{ICH}$, we further evaluated the MMP-9 levels in the co-culture medium by ELISA. We found that MMP-9 expression was significantly increased in the ICH group (Supplementary Figure 1) and did not show many alterations under CYC treatment. The multiple factors secreted in the co-culture medium eventually affect the expression of TJs protein and permeability of $\mathrm{BBB}$. Other mechanisms that may affect the BBB integrity in the bEnd3 astrocytes co-culture system still needs experiments in our further study. Also, we found that compared with the co-culture with $\mathrm{ICH}$-induced astrocytes and vehicle, CYC treatment caused a remarkable decrease in the expressions of ZO-1 and claudin-5 in bEnd 3 cells, indicating a protective role of astrocytic $\mathrm{SHH}$ signaling pathway on $\mathrm{ICH}$ by modulating TJs expression.

The SHH pathway is a critical signaling pathway in a variety of diseases, such as asthma, gastrointestinal system, preeclampsia, bladder cancer, and brain injury (Wang et al., 2008, 2019; Amankulor et al., 2009; Sims et al., 2009; Syed et al., 2016; Hu et al., 2017; Qi et al., 2018; Liao et al., 2019). The potential biological mechanisms of the $\mathrm{SHH}$ signaling pathway in CNS may be associated with a wide range of pathological processes, such as neurogenesis, anti-oxidation, autophagy, and antiapoptosis. Several types of research demonstrated that the $\mathrm{SHH}$ 
A

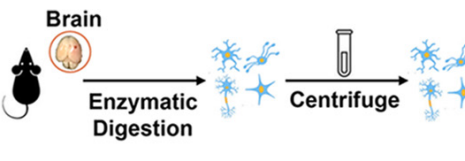

B

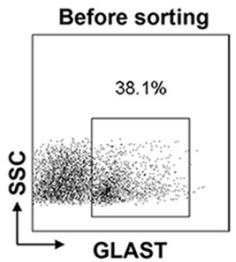

C

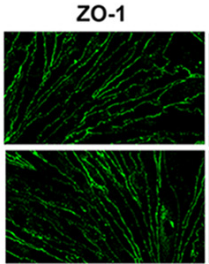

ICH+Vehicle
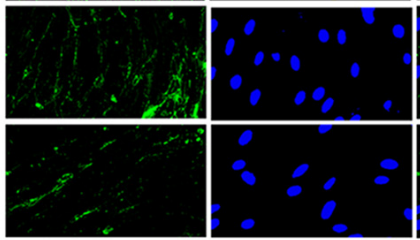

E

Claudin-5

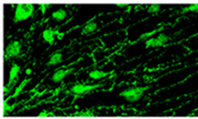

bEnd3

SHAM

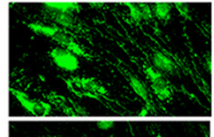

$\mathrm{ICH}+$ Vehicle

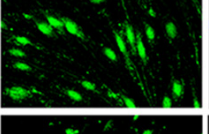

$\mathrm{ICH}+\mathrm{CYC}$
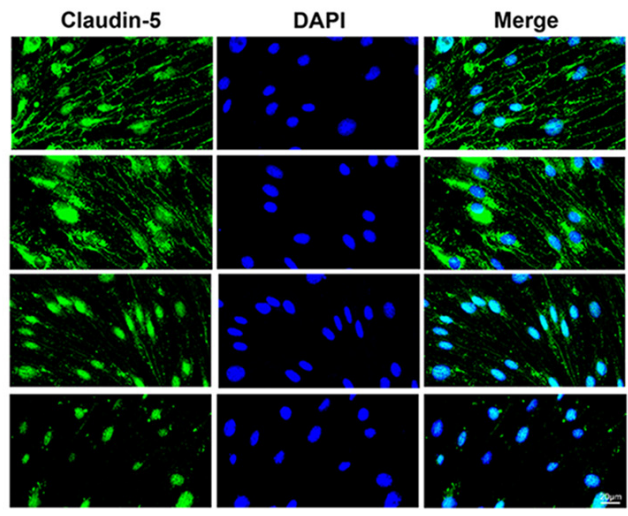

G

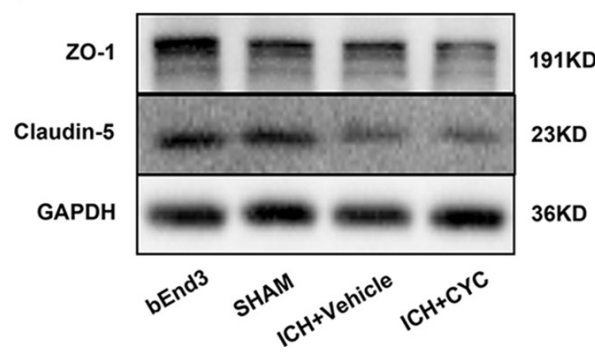

D

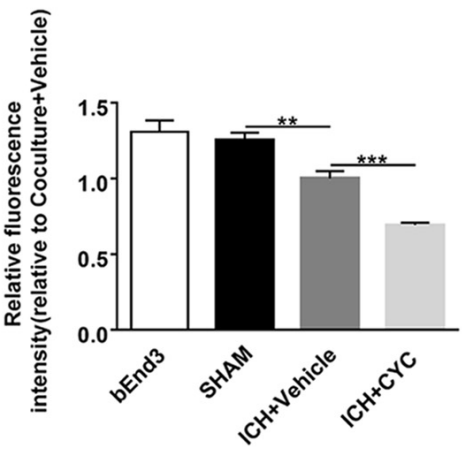

F
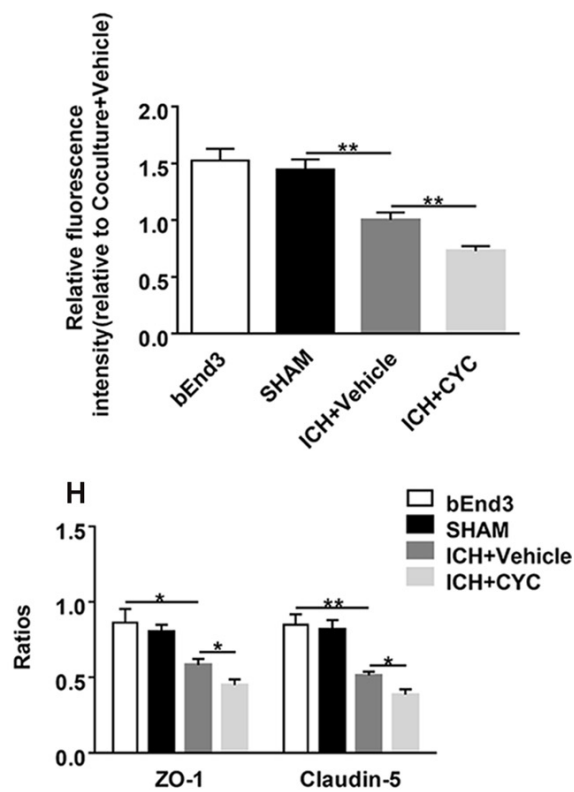

FIGURE 5 | Astrocytic SHH signaling pathway regulates the expressions of TJs in astrocyte-endothelial co-culture in vitro. ICH was induced in mice by injection of autologous blood. (A,B) Procedures for the isolation and purification of astrocytes (GLAST+) from sham and ICH mice brains. On day 3 after surgery, brain tissues of mice were harvested. (C-F) Representative images and quantification of fluorescence intensity showed the expressions of ZO-1 and claudin-5 in bEnd3 cells that were cultured alone, co-cultured with sham-induced astrocytes, co-cultured with $\mathrm{ICH}$-induced astrocytes and vehicle, and co-cultured with ICH-induced astrocytes and CYC. $n=6$ mice per group. (G,H) Western blot images showed the expressions of ZO-1 and claudin- 5 in bEnd3 cells that were cultured alone, co-cultured with sham-induced astrocytes, co-cultured with ICH-induced astrocytes and vehicle, and co-cultured with ICH-induced astrocytes and CYC. $n=6$ mice per group. Data are presented as mean \pm SEM. ${ }^{*} P<0.05,{ }^{\star \star} P<0.01,{ }^{\star \star \star} P<0.001$. 

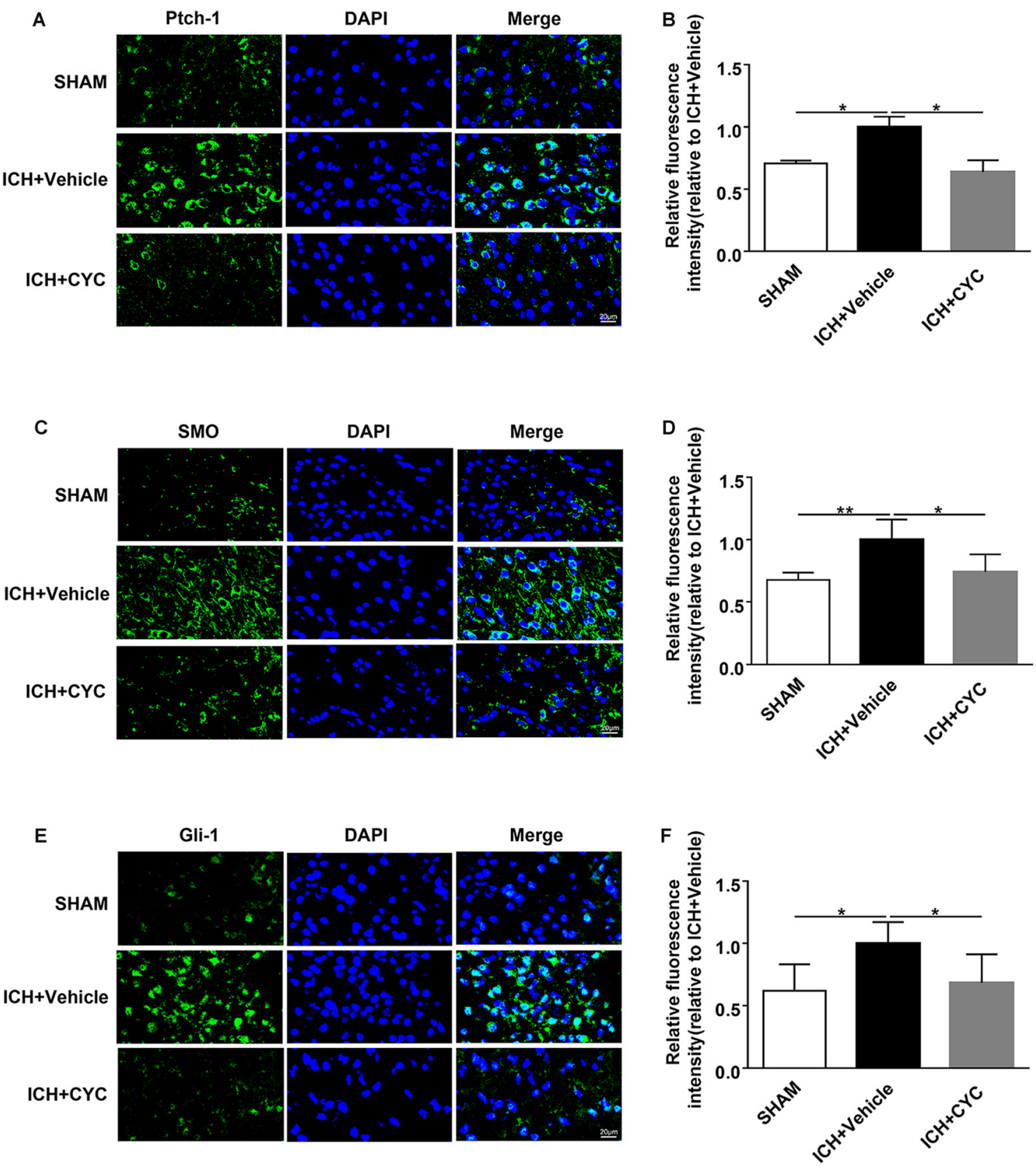

FIGURE 6 | CYC inhibits the activation of the SHH-Gli-1 axis in ICH mice. ICH was induced in mice by injection of collagenase. Mice received $\mathrm{CYC}$ (10 mg/kg) or an equal volume of the vehicle via intraperitoneal injection once a day, starting from day 0 to day 2 after sham or ICH operations. On day 3 , brain samples were prepared and the expressions of the downstream molecules of the $\mathrm{SHH}-\mathrm{Gli}-1$ axis were measured by immunostaining. (A,B) Representative images and quantification of fluorescence intensity showed the expressions of Ptch-1 in mice received $\mathrm{CYC}$ treatment or vehicle on day 3 after ICH, $n=3$ mice per group. (C,D) Representative images and quantification of fluorescence intensity showed the expressions of SMO in mice received CYC treatment or vehicle on day 3 after $\mathrm{ICH}, n=5$ mice per group. (E,F) Representative images and quantification of fluorescence intensity showed the expressions of Gli- 1 in mice received CYC treatment or vehicle on day 3 after $\mathrm{ICH}, n=5$ mice per group. Data are presented as mean \pm SEM. ${ }^{\star} P<0.05,{ }^{\star \star} P<0.01$.

signaling pathway may be involved in the integrity of BBB and the endogenous anti-inflammatory response to CNS immune attacks (Alvarez et al., 2011; Singh et al., 2016; Zhen et al., 2018). We appreciate these thoughtful comments. Li et al. (2013) reported the role of the $\mathrm{SHH}$ signaling pathway in the experimental SAH model, demonstrating that the SHH signaling pathway could be involved in the pathogenesis of $\mathrm{SAH}$, suggesting possible protective effects on the SAH-induced oxidative stress by the PI3K/Akt pathway. Different from the SAH, which mainly affect the subarachnoid space, ICH showed a marked increase in BBB hyperpermeability, brain edema, and neurological deficit after bleeding into the brain parenchyma (Smith and Eskey, 


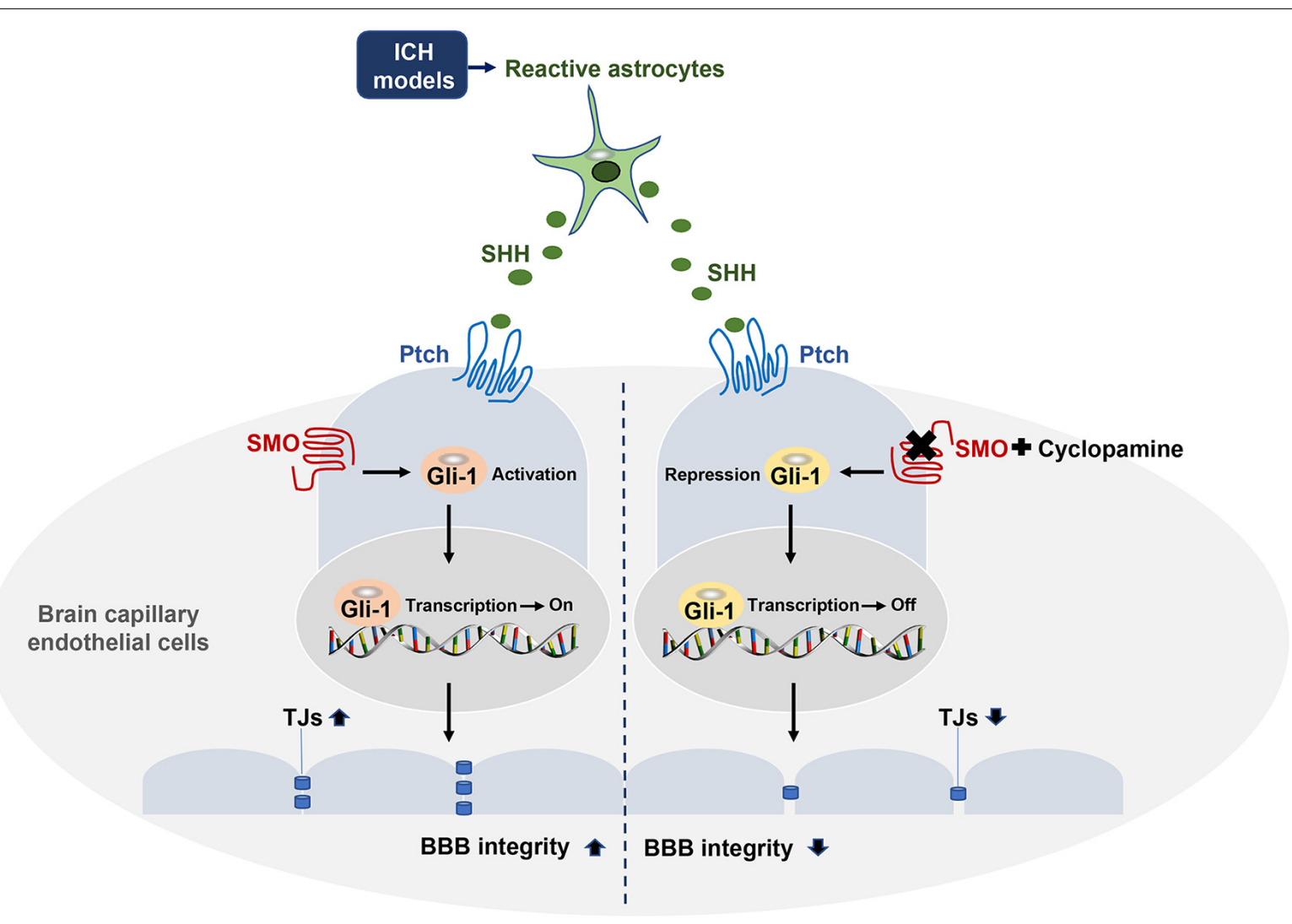

FIGURE 7 | Astrocytic SHH signaling pathway regulates BBB integrity after ICH. SHH secreted by astrocytes binds to its receptor Ptch-1 in endothelial cells (ECs), leading to the activation of SMO and its downstream transcription factor Gli-1. Activated Gli-1 upregulates TJs and enhances BBB integrity. Upon the binding of $\mathrm{SMO}$ to $\mathrm{CYC}$, this pathway is repressed, resulting in decreased TJs expressions and exacerbated BBB permeability.

2011). Thus, the possible contribution of the SHH signaling pathway on ICH has been elucidated in our study, clarifying the protective effect of the astrocytic $\mathrm{SHH}$ signaling pathway on $\mathrm{ICH}$ by TJs regulation. Our study is the first to show that the inhibition of the $\mathrm{SHH}$ signaling pathway by CYC significantly aggravates $\mathrm{ICH}$-induced neurological dysfunction, brain edema, hematoma volume, and BBB permeability in mice. Gli-1 is a crucial regulator of $\mathrm{BBB}$ integrity and TJs expressions. As an SMO antagonist, CYC can selectively bind to SMO, ultimately lead to the repression of Gli-1 transcription factors into the nucleus, resulting in lower TJs expressions in ECs throughout the ICH-induced damages in vivo and in vitro. The potential mechanism may involve the regulation of TJs expressions in ECs through the SHH-Gli-1 axis, as shown in Figure 7. Our study demonstrates that $\mathrm{SHH}$ might be used as a target to protect $\mathrm{BBB}$ integrity for early treatment in $\mathrm{ICH}$.

SHH signaling pathway has been considered as vital for BBB function via TJs regulation. In our study, the $\mathrm{SHH}-\mathrm{Gli}$ axis was shown to be essential for $\mathrm{BBB}$ integrity following $\mathrm{ICH}$, since inhibition of $\mathrm{SHH}$ led to severer $\mathrm{BBB}$ damage. However, it has been reported that $\mathrm{SHH}$-Ptch also contributes to upregulate vascular endothelial grow factor (VEGF), angiopoietins Ang-1 and Ang-2, which participate in the development of vasculature ingrowth and BBB function (Pola et al., 2001). Thus, SHH may play a protective role in BBB integrity also by promoting VEGF, Ang-1, and Ang-2 production. Thus, the SMO-Gli-1-TJ relation in the protective role of astrocytic $\mathrm{SHH}$ in $\mathrm{BBB}$ integrity still needs further investigation. In our study, further experiments also are needed to clarify the protective role of astrocyte-derived $\mathrm{SHH}$ in $\mathrm{ICH}$ (i.e., a transgenic mouse line in which the $\mathrm{SHH}$ is overexpressed or knocked down in astrocytes). Furthermore, the agonist of the SHH signaling pathway may be used to identify the potential therapeutic property of the SHH signaling pathway in $\mathrm{ICH}$.

\section{CONCLUSION}

Our results show that the astrocytic $\mathrm{SHH}$ signaling pathway plays a pivotal role in the protection of BBB integrity and function in $\mathrm{ICH}$, suggesting the potential therapeutic value of $\mathrm{SHH}$ in ameliorating ICH injury. The underlying mechanism of astrocytic SHH-targeting therapy needs to be further studied.

\section{DATA AVAILABILITY STATEMENT}

All datasets presented in this study are included in the article/Supplementary Material. 


\section{ETHICS STATEMENT}

All experiments were approved by the Animal Experiments Ethical Committee of Tianjin Medical University General Hospital and Jining Medical University (the number of the approval of ethics statement: 2019-FY-024). This study was managed following the National Institutes of Health Guide for the Care and Use of Laboratory Animals in China.

\section{AUTHOR CONTRIBUTIONS}

WJ and NZ formulated the concept and designed the study. GX, $\mathrm{TZ}$, and DL performed the studies. XZ, HL, and XL analyzed the data and interpreted the results. PC contributed new reagents or analytic tools. GX, TZ, and ML wrote and revised the article. All authors contributed to the article and approved the submitted version.

\section{REFERENCES}

Alvarez, J. I., Dodelet-Devillers, A., Kebir, H., Ifergan, I., Fabre, P. J., Terouz, S., et al. (2011). The Hedgehog pathway promotes blood-brain barrier integrity and CNS immune quiescence. Science 334, 1727-1731. doi: 10.1126/science. 1206936

Alvarez, J. I., Katayama, T., and Prat, A. (2013). Glial influence on the blood brain barrier. Glia 61, 1939-1958. doi: 10.1002/glia.22575

Amankulor, N. M., Hambardzumyan, D., Pyonteck, S. M., Becher, O. J., Joyce, J. A., and Holland, E. C. (2009). Sonic hedgehog pathway activation is induced by acute brain injury and regulated by injury-related inflammation. J. Neurosci. 29, 10299-10308. doi: 10.1523/JNEUROSCI.250009.2009

Arthur, F. E., Shivers, R. R., and Bowman, P. D. (1987). Astrocyte-mediated induction of tight junctions in brain capillary endothelium: an efficient in vitro model. Brain Res. 433, 155-159. doi: 10.1016/0165-3806(87)90075-7

Bohannon, D. G., Ko, A., Filipowicz, A. R., Kuroda, M. J., and Kim, W. K. (2019). Dysregulation of sonic hedgehog pathway and pericytes in the brain after lentiviral infection. J. Neuroinflammation 16:86. doi: 10.1186/s12974-0191463-y

Brown, R. C., Morris, A. P., and O’Neil, R. G. (2007). Tight junction protein expression and barrier properties of immortalized mouse brain microvessel endothelial cells. Brain Res. 1130, 17-30. doi: 10.1016/j.brainres.2006.10.083

Caradu, C., Guy, A., James, C., Reynaud, A., Gadeau, A. P., and Renault, M. A. (2018). Endogenous Sonic Hedgehog limits inflammation and angiogenesis in the ischaemic skeletal muscle of mice. Cardiovasc Res. 114, 759-770. doi: $10.1093 / \mathrm{cvr} / \mathrm{cvy} 017$

Cazet, A. S., Hui, M. N., Elsworth, B. L., Wu, S. Z., Roden, D., Chan, C.-L., et al. (2018). Targeting stromal remodeling and cancer stem cell plasticity overcomes chemoresistance in triple negative breast cancer. Nat. Commun. 9:2897. doi: 10.1038/s41467-018-05220-6

Chen, J. K., Taipale, J., Cooper, M. K., and Beachy, P. A. (2002). Inhibition of Hedgehog signaling by direct binding of cyclopamine to Smoothened. Genes Dev. 16, 2743-2748. doi: 10.1101/gad.1025302

Chen, S. D., Yang, J. L., Hwang, W. C., and Yang, D. I. (2018). Emerging roles of sonic hedgehog in adult neurological diseases: neurogenesis and beyond. Int. J. Mol. Sci. 19:2423. doi: 10.3390/ijms19082423

Chiu, C. D., Yao, N. W., Guo, J. H., Shen, C. C., Lee, H. T., Chiu, Y. P., et al. (2017). Inhibition of astrocytic activity alleviates sequela in acute stages of intracerebral hemorrhage. Oncotarget 8, 94850-94861. doi: 10.18632/oncotarget.22022

Cooper, M. K., Porter, J. A., Young, K. E., and Beachy, P. A. (1998). Teratogenmediated inhibition of target tissue response to Shh signaling. Science 280, 1603-1607. doi: 10.1126/science.280.5369.1603

Dennis, M. S., Burn, J. P., Sandercock, P. A., Bamford, J. M., Wade, D. T., and Warlow, C. P. (1993). Long-term survival after first-ever stroke: the

\section{FUNDING}

This study was supported by the National Natural Science Foundation of China (81801197, 81870954 to WJ and 81901228 to DL) and Natural Science Foundation of Tianjin (19JCQNJC10500 to WJ and 20JCYBJC00400 to ML).

\section{ACKNOWLEDGMENTS}

We appreciate the editorial assistance from Kristofer Wood. We thank Dr. Junwei Hao for the help in the study design and manuscript drafting.

\section{SUPPLEMENTARY MATERIAL}

The Supplementary Material for this article can be found online at: https://www.frontiersin.org/articles/10.3389/fncel.2020.5756 90/full\#supplementary-material.

Oxfordshire Community Stroke Project. Stroke 24, 796-800. doi: 10.1161/01. str.24.6.796

Gaillard, P. J., Voorwinden, L. H., Nielsen, J. L., Ivanov, A., Atsumi, R., Engman, H., et al. (2001). Establishment and functional characterization of an in vitro model of the blood-brain barrier, comprising a co-culture of brain capillary endothelial cells and astrocytes. Eur. J. Pharm. Sci. 12, 215-222. doi: 10.1016/s0928-0987(00)00123-8

Grossetete, M., and Rosenberg, G. A. (2008). Matrix metalloproteinase inhibition facilitates cell death in intracerebral hemorrhage in mouse. J. Cereb. Blood Flow Metab. 28, 752-763. doi: 10.1038/sj.jcbfm.9600572

Han, R., Luo, J., Shi, Y., Yao, Y., and Hao, J. (2017). PD-L1 (programmed death ligand 1) protects against experimental intracerebral hemorrhage-induced brain injury. Stroke 48, 2255-2262. doi: 10.1161/STROKEAHA.117.016705

He, W., Cui, L., Zhang, C., Zhang, X., He, J., Xie, Y., et al. (2017). Sonic hedgehog promotes neurite outgrowth of cortical neurons under oxidative stress: Involving of mitochondria and energy metabolism. Exp. Cell Res. 350, 83-90. doi: 10.1016/j.yexcr.2016.11.008

He, Q. W., Xia, Y. P., Chen, S. C., Wang, Y., Huang, M., Huang, Y., et al. (2013). Astrocyte-derived sonic hedgehog contributes to angiogenesis in brain microvascular endothelial cells via RhoA/ROCK pathway after oxygen-glucose deprivation. Mol. Neurobiol. 47, 976-987. doi: 10.1007/s12035-013-8396-8

Hu, Q., Li, T., Wang, L., Xie, Y., Liu, S., Bai, X., et al. (2017). Neuroprotective effects of a smoothened receptor agonist against early brain injury after experimental subarachnoid hemorrhage in rats. Front. Cell. Neurosci. 10:306. doi: 10.3389 /fncel.2016.00306

Irvine, D. A., Zhang, B., Kinstrie, R., Tarafdar, A., Morrison, H., Campbell, V. L., et al. (2016). Deregulated hedgehog pathway signaling is inhibited by the smoothened antagonist LDE225 (Sonidegib) in chronic phase chronic myeloid leukaemia. Sci. Rep. 6:25476. doi: 10.1038/srep25476

Isobe, I., Watanabe, T., Yotsuyanagi, T., Hazemoto, N., Yamagata, K., Ueki, T., et al. (1996). Astrocytic contributions to blood-brain barrier (BBB) formation by endothelial cells: a possible use of aortic endothelial cell for in vitro BBB model. Neurochem. Int. 28, 523-533. doi: 10.1016/0197-0186(95)00142-5

Janzer, R. C., and Raff, M. C. (1987). Astrocytes induce blood-brain barrier properties in endothelial cells. Nature 325, 253-257. doi: 10.1038/325253a0

Jin, W. N., Shi, S. X., Li, Z., Li, M., Wood, K., Gonzales, R. J., et al. (2017). Depletion of microglia exacerbates post ischemic inflammation and brain injury. J. Cereb. Blood Flow Metab. 37, 2224-2236. doi: 10.3389/fimmu.2018.01696

Keep, R. F., Andjelkovic, A. V., Xiang, J., Stamatovic, S. M., Antonetti, D. A., Hua, Y., et al. (2018). Brain endothelial cell junctions after cerebral hemorrhage: changes, mechanisms and therapeutic targets. J. Cereb. Blood Flow Metab. 38, 1255-1275. doi: 10.1177/0271678X18774666

Keep, R. F., Zhou, N., Xiang, J., Andjelkovic, A. V., Hua, Y., and Xi, G. (2014). Vascular disruption and blood-brain barrier dysfunction in intracerebral hemorrhage. Fluids Barriers CNS 11:18. doi: 10.1186/2045-8118-11-18 
Laird, M. D., Sukumari-Ramesh, S., Swift, A. E., Meiler, S. E., Vender, J. R., and Dhandapani, K. M. (2010). Curcumin attenuates cerebral edema following traumatic brain injury in mice: a possible role for aquaporin-4? J. Neurochem. 113, 637-648. doi: 10.1111/j.1471-4159.2010.06630.x

Lauressergues, E., Heusler, P., Lestienne, F., Troulier, D., Rauly-Lestienne, I., Tourette, A., et al. (2016). Pharmacological evaluation of a series of smoothened antagonists in signaling pathways and after topical application in a depilated mouse model. Pharmacol. Res. Perspect. 4:e00214. doi: 10.1002/ prp2.214

Li, D., Lang, W., Zhou, C., Wu, C., Zhang, F., Liu, Q., et al. (2018). Upregulation of microglial ZEB1 ameliorates brain damage after acute ischemic stroke. Cell Rep. 22, 3574-3586. doi: 10.1016/j.celrep.2018.03.011

Li, M., Li, Z., Ren, H., Jin, W. N., Wood, K., Liu, Q., et al. (2017a). Colony stimulating factor 1 receptor inhibition eliminates microglia and attenuates brain injury after intracerebral hemorrhage. J. Cereb. Blood Flow Metab. 37, 2383-2395. doi: 10.1177/0271678X16666551

Li, M., Li, Z., Yao, Y., Jin, W. N., Wood, K., Liu, Q., et al. (2017b). Astrocytederived interleukin-15 exacerbates ischemic brain injury via propagation of cellular immunity. Proc. Natl. Acad. Sci. U S A 114, E396-E405. doi: 10.1073/pnas.1612930114

Li, M., Ren, H., Sheth, K. N., Shi, F. D., and Liu, Q. (2017c). A TSPO ligand attenuates brain injury after intracerebral hemorrhage. FASEB J. 31, 3278-3287. doi: 10.1096/fj.201601377RR

Li, H. D., Li, M., Shi, E., Jin, W. N., Wood, K., Gonzales, R., et al. (2017). A translocator protein $18 \mathrm{kDa}$ agonist protects against cerebral ischemia/reperfusion injury. J. Neuroinflammation 14:151. doi: 10.1186/s12974-017-0921-7

Li, X., Ma, Q., Xu, Q., Liu, H., Lei, J., Duan, W., et al. (2012). SDF1/CXCR4 signaling induces pancreatic cancer cell invasion and epithelialmesenchymal transition in vitro through non-canonical activation of Hedgehog pathway. Cancer Lett. 322, 169-176. doi: 10.1016/j.canlet.2012. 02.035

Li, G., Simon, M. J., Cancel, L. M., Shi, Z. D., Ji, X., Tarbell, J. M., et al. (2010). Permeability of endothelial and astrocyte cocultures: in vitro blood-brain barrier models for drug delivery studies. Ann. Biomed. Eng. 38, 2499-2511. doi: 10.1007/s10439-010-0023-5

Li, T., Zhang, J., Liu, R. Y., Lian, Z. G., Chen, X. L., Ma, L., et al. (2013). The role of the sonic hedgehog signaling pathway in early brain injury after experimental subarachnoid hemorrhage in rats. Neurosci. Lett. 552, 81-86. doi: 10.1016/j. neulet.2013.07.042

Liao, Q. P., Xu, Q., and Yan, J. Y. (2019). SHH expression in placental tissues and trophoblast cell oxidative stress injury during preeclampsia. Eur. Rev. Med. Pharmacol. Sci. 23, 6026-6034. doi: 10.26355/eurrev_201907_18415

Madangarli, N., Bonsack, F., Dasari, R., and Sukumari-Ramesh, S. (2019). Intracerebral hemorrhage: blood components and neurotoxicity. Brain Sci. 9:316. doi: 10.3390/brainsci9110316

Michinaga, S., and Koyama, Y. (2019). Dual roles of astrocyte-derived factors in regulation of blood-brain barrier function after brain damage. Int. J. Mol. Sci. 20:571. doi: $10.3390 /$ ijms 20030571

Min, H., Hong, J., Cho, I.-H., Jang, Y. H., Lee, H., Kim, D., et al. (2015). TLR2induced astrocyte MMP9 activation compromises the blood brain barrier and exacerbates intracerebral hemorrhage in animal models. Mol. Brain 8:23. doi: 10.1186/s13041-015-0116-Z

Moriguchi, S., Yabuki, Y., and Fukunaga, K. (2012). Reduced calcium/calmodulindependent protein kinase II activity in the hippocampus is associated with impaired cognitive function in MPTP-treated mice. J. Neurochem. 120, 541-551. doi: 10.1111/j.1471-4159.2011.07608.x

Obermeier, B., Daneman, R., and Ransohoff, R. M. (2013). Development, maintenance and disruption of the blood-brain barrier. Nat. Med. 19, 1584-1596. doi: 10.1038/nm.3407

Palma, V., Lim, D. A., Dahmane, N., Sánchez, P., Brionne, T. C., Herzberg, C. D., et al. (2005). Sonic hedgehog controls stem cell behavior in the postnatal and adult brain. Development 132, 335-344. doi: 10.1242/dev.01567

Pan, S., Wu, X., Jiang, J., Gao, W., Wan, Y., Cheng, D., et al. (2010). Discovery of NVP-LDE225, a potent and selective smoothened antagonist. ACS Med. Chem. Lett. 1, 130-134. doi: 10.1021/ml1000307

Pola, R., Ling, L. E., Silver, M., Corbley, M. J., Kearney, M., Blake Pepinsky, R., et al. (2001). The morphogen Sonic hedgehog is an indirect angiogenic agent upregulating two families of angiogenic growth factors. Nat. Med. 7, 706-711. doi: $10.1038 / 89083$

Qi, Y., Zhao, W., Wang, Z., Xie, Q., Cao, J., and Meng, X. (2018). Cross regulation of signaling pathways in gastrointestinal stromal tumor. Oncol. Lett. 16, 6770-6776. doi: 10.3892/ol.2018.9494

Qureshi, A. I., Mendelow, A. D., and Hanley, D. F. (2009). Intracerebral haemorrhage. Lancet 373, 1632-1644. doi: 10.1016/S0140-6736(09)60371-8

Qureshi, A. I., Tuhrim, S., Broderick, J. P., Batjer, H. H., Hondo, H., and Hanley, D. F. (2001). Spontaneous intracerebral hemorrhage. N. Engl. J. Med. 344, 1450-1460. doi: 10.1056/NEJM200105103441907

Ren, H., Kong, Y., Liu, Z., Zang, D., Yang, X., Wood, K., et al. (2018). Selective NLRP3 (pyrin domain-containing protein 3) inflammasome inhibitor reduces brain injury after intracerebral hemorrhage. Stroke 49, 184-192. doi: 10.1161/STROKEAHA.117.018904

Rimkus, T. K., Carpenter, R. L., Qasem, S., Chan, M., and Lo, H. W. (2016). Targeting the sonic hedgehog signaling pathway: review of smoothened and gli inhibitors. Cancers 8:22. doi: 10.3390/cancers8020022

Rynkowski, M. A., Kim, G. H., Komotar, R. J., Otten, M. L., Ducruet, A. F., Zacharia, B. E., et al. (2008). A mouse model of intracerebral hemorrhage using autologous blood infusion. Nat. Protoc. 3, 122-128. doi: 10.1038/nprot. 2007.513

Scheuer, T., Klein, L. S., Bührer, C., Endesfelder, S., and Schmitz, T. (2019). Transient improvement of cerebellar oligodendroglial development in a neonatal hyperoxia model by PDGFA treatment. Dev. Neurobiol. 79, 222-235. doi: 10.1002/dneu.22667

Sims, J. R., Lee, S. W., Topalkara, K., Qiu, J., Xu, J., Zhou, Z., et al. (2009). Sonic hedgehog regulates ischemia/hypoxia-induced neural progenitor proliferation. Stroke 40, 3618-3626. doi: 10.1161/STROKEAHA.109.561951

Singh, V. B., Singh, M. V., Gorantla, S., Poluektova, L. Y., and Maggirwar, S. B. (2016). Smoothened agonist reduces human immunodeficiency virus type-1induced blood-brain barrier breakdown in humanized mice. Sci. Rep. 6:26876. doi: $10.1038 /$ srep26876

Sirko, S., Behrendt, G., Johansson, P. A., Tripathi, P., Costa, M., Bek, S., et al. (2013). Reactive glia in the injured brain acquire stem cell properties in response to sonic hedgehog. Cell Stem Cell 12, 426-439doi: 10.1016/j.stem. 2013.01.019

Smith, S. D., and Eskey, C. J. (2011). Hemorrhagic stroke. Radiol. Clin. North Am. 49, 27-45. doi: 10.1016/j.rcl.2010.07.011

Sukumari-Ramesh, S., Alleyne, C. H. Jr., and Dhandapani, K. M. (2012). Astrogliosis: a target for intervention in intracerebral hemorrhage? Transl. Stroke Res. 3, 80-87. doi: 10.1007/s12975-012-0165-x

Sun, M., Zhang, N., Wang, X., Li, Y., Qi, W., Zhang, H., et al. (2016). Hedgehog pathway is involved in nitidine chloride induced inhibition of epithelialmesenchymal transition and cancer stem cells-like properties in breast cancer cells. Cell Biosci. 6:44. doi: 10.1186/s13578-016-0104-8

Syed, I. S., Pedram, A., and Farhat, W. A. (2016). Role of sonic hedgehog (Shh) signaling in bladder cancer stemness and tumorigenesis. Curr. Urol. Rep. 17:11. doi: 10.1007/s11934-015-0568-9

Tschoe, C., Bushnell, C. D., Duncan, P. W., Alexander-Miller, M. A., and Wolfe, S. Q. (2020). Neuroinflammation after intracerebral hemorrhage and potential therapeutic targets. J. Stroke 22, 29-46. doi: 10.5853/jos.2019.02236

Wang, Y., Imitola, J., Rasmussen, S., O'Connor, K. C., and Khoury, S. J. (2008). Paradoxical dysregulation of the neural stem cell pathway sonic hedgehog-Gli1 in autoimmune encephalomyelitis and multiple sclerosis. Ann. Neurol. 64, 417-427. doi: 10.1002/ana.21457

Wang, Y., Jin, S., Sonobe, Y., Cheng, Y., Horiuchi, H., Parajuli, B., et al. (2014). Interleukin-1 $\beta$ induces blood-brain barrier disruption by downregulating Sonic hedgehog in astrocytes. PLoS One 9:e110024. doi: 10.1371/journal.pone. 0110024

Wang, X.-Z., Zhang, H.-H., Qian, Y.-L., and Tang, L.-F. (2019). Sonic hedgehog (Shh) and CC chemokine ligand 2 signaling pathways in asthma. J. Chin. Med. Assoc. 82, 343-350. doi: 10.1097/JCMA.0000000000000094

Xi, G., Strahle, J., Hua, Y., and Keep, R. F. (2013). Progress in translational research on intracerebral hemorrhage: is there an end in sight? Prog. Neurobiol. 115, 45-63. doi: 10.1016/j.pneurobio.2013.09.007

Yang, H., Cong, W.-N., Yoon, J. S., and Egan, J. M. (2015). Vismodegib, an antagonist of hedgehog signaling, directly alters taste molecular signaling in taste buds. Cancer Med. 4, 245-252. doi: 10.1002/cam4.350 
Yang, X., Ren, H., Wood, K., Li, M., Qiu, S., Shi, F. D., et al. (2018). Depletion of microglia augments the dopaminergic neurotoxicity of MPTP. FASEB J. 32, 3336-3345. doi: 10.1096/fj.201700833RR

Yu, Q.-J., Tao, H., Wang, X., and Li, M.-C. (2015). Targeting brain microvascular endothelial cells: a therapeutic approach to neuroprotection against stroke. Neural Regen. Res. 10, 1882-1891. doi: 10.4103/1673-5374. 170324

Zhang, Y., Zhang, X., Cui, L., Chen, R., Zhang, C., Li, Y., et al. (2017). Salvianolic Acids for Injection (SAFI) promotes functional recovery and neurogenesis via sonic hedgehog pathway after stroke in mice. Neurochem. Int. 110, 38-48. doi: 10.1016/j.neuint.2017.09.001

Zhen, H., Zhao, L., Ling, Z., Kuo, L., Xue, X., and Feng, J. (2018). Wip1 regulates blood-brain barrier function and neuro-inflammation induced by lipopolysaccharide via the sonic hedgehog signaling signaling pathway. Mol. Immunol. 93, 31-37. doi: 10.1016/j.molimm.2017. 09.020

Conflict of Interest: The authors declare that the research was conducted in the absence of any commercial or financial relationships that could be construed as a potential conflict of interest.

Copyright (๐ 2020 Xing, Zhao, Zhang, Li, Li, Cui, Li, Li, Zhang and Jiang. This is an open-access article distributed under the terms of the Creative Commons Attribution License (CC BY). The use, distribution or reproduction in other forums is permitted, provided the original author(s) and the copyright owner(s) are credited and that the original publication in this journal is cited, in accordance with accepted academic practice. No use, distribution or reproduction is permitted which does not comply with these terms. 